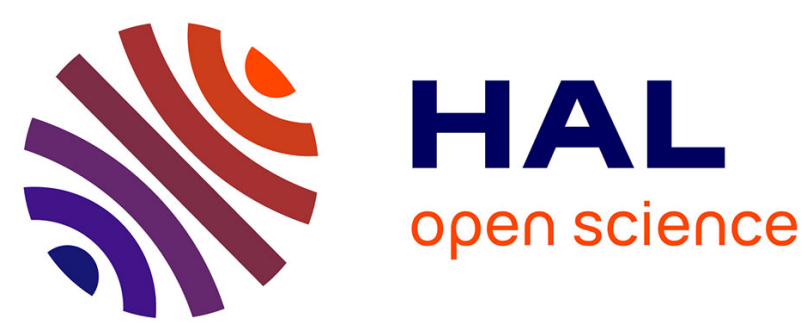

\title{
Ten Facets, One Force Field: The GAL19 Force Field for Water-Noble Metal Interfaces
}

Paul Clabaut, Paul Fleurat-Lessard, Carine Michel, Stephan N. Steinmann

\section{To cite this version:}

Paul Clabaut, Paul Fleurat-Lessard, Carine Michel, Stephan N. Steinmann. Ten Facets, One Force Field: The GAL19 Force Field for Water-Noble Metal Interfaces. Journal of Chemical Theory and Computation, 2020, 16 (7), pp.4565-4578. 10.1021/acs.jctc.0c00091 . hal-03424265

\section{HAL Id: hal-03424265 https://hal.science/hal-03424265}

Submitted on 10 Nov 2021

HAL is a multi-disciplinary open access archive for the deposit and dissemination of scientific research documents, whether they are published or not. The documents may come from teaching and research institutions in France or abroad, or from public or private research centers.
L'archive ouverte pluridisciplinaire HAL, est destinée au dépôt et à la diffusion de documents scientifiques de niveau recherche, publiés ou non, émanant des établissements d'enseignement et de recherche français ou étrangers, des laboratoires publics ou privés. 


\title{
Ten Facets, One Force Field: The GAL19
}

\section{Force Field for Water - Noble Metal Interfaces}

\author{
Paul Clabaut, ${ }^{\dagger}$ Paul Fleurat-Lessard ${ }^{\ddagger}{ }^{\text {Carine Michel, }}{ }^{\dagger}$ and Stephan N. Steinmann*,† \\ †Univ Lyon, Ecole Normale Supérieure de Lyon, CNRS Université Lyon 1, Laboratoire de Chimie \\ UMR 5182, 46 allée d'Italie, F-69364, LYON, France \\ $\ddagger$ Université de Bourgogne Franche-Comté (UBFC), Institut de Chimie Moléculaire de l'Université \\ de Bourgogne (ICMUB), UMR CNRS 6302, 9 avenue Alain Savary 21078 Dijon, France \\ E-mail: stephan.steinmann@ens-lyon.fr
}

Phone: (+33)4 72728155

\begin{abstract}
Understanding the structure of the water/metal interfaces plays an important role in many areas ranging from surface chemistry to environmental processes. The size, required phase-space sampling and the slow diffusion of molecules at the water/metal interfaces motivate the development of accurate force-fields. We develop and parametrize GAL19, a novel force-field to describe the interaction of water with two facets (111 and 100) of five metals ( $\mathrm{Pt}, \mathrm{Pd}, \mathrm{Au}, \mathrm{Ag}, \mathrm{Cu})$. To increase transferability compared to its predecessor GAL17, the water-metal interaction is described as a sum of pair-wise terms. The interaction energy has three contributions: (i) physisorption is described via a Tang and Toennies potential, (ii) chemisorption and surface corrugation relies on an attractive Gaussian term and (iii) the angular dependence is explicitly included as a truncated Fourier series. 13 parameters are used for each metal surface and were fitted on 250 water adsorption energies computed at the PBE+dDsC level.
\end{abstract}


The performance of GAL19 was evaluated on a set of more than 600 DFT adsorption energies for each surface, leading to an average root mean square deviation (RMSD) of only $1 \mathrm{kcal} / \mathrm{mol}$, correctly reproducing the adsorption trends: strong on $\mathrm{Pt}$ and $\mathrm{Pd}$ but weaker on $\mathrm{Ag}, \mathrm{Au}$ and $\mathrm{Cu}$. This force-field was then used to simulate the water/metal interface for all ten surfaces for $1 \mathrm{~ns}$. Structural analyses reveal similar tendencies for all surfaces: a first, dense water layer that is mostly adsorbed on the metal top sites, and a second layer up to around $6 \AA$, which is less structured. On Pt and $\mathrm{Pd}$, the first layer is strongly organized with water lying flat on the surface. The pairwise additive functional form allows to simulate the water adsorption on alloys, which is demonstrated at the example of $\mathrm{Ag} / \mathrm{Cu}$ and $\mathrm{Au} / \mathrm{Pt}$ alloys. The water/Ag-Cu interface is predicted to be disordered with water mostly adsorbed on $\mathrm{Cu}$ which should exacerbate the Ag reactivity. On the contrary, incorporating Pt into Au materials leads to a structuring of the water interface. Our promising results make GAL19 an ideal candidate to get representative sampling of complex metal/water interfaces as a first step towards accurate estimation of free energies of reactions in solution at the metal interface.

\section{Introduction}

The metal/water interface is key in many technologically relevant systems, ranging from heterogeneous (electro-)catalysis ${ }^{1,2}$ to tribology ${ }^{3}$ and corrosion. ${ }^{4}$ Beyond the prototypical monometallic surfaces, alloys are of key importance in the domain of catalysis ${ }^{5,6}$ and active research in corrosion. ${ }^{7}$ The atomistic understanding of metal/liquid interfaces remains poor, despite experimental ${ }^{8-10}$ and computational ${ }^{11-24}$ efforts throughout the last 30 years, exclusively devoted to monometallic single-crystal surfaces. The origin of the difficulties to characterize the metal/water interface at an atomistic level comes from its non-crystallinity. In absence of a long-range order, only indirect spectroscopic evidence is available regarding the organization of water at metallic interfaces. Similarly, from a 
computational perspective, the amorphous nature of the interface requires large simulation cells in combination with thorough phase-space sampling due to the slow equilibration at the interface that features strong interactions with the water molecules. ${ }^{18,21}$

Large simulation cells and extensive phase-space sampling is routinely applied for bio-molecules relying on molecular mechanics (MM), i.e., simple force fields, instead of evaluating the energies and forces from first-principles. These force fields have been optimized over the last sixty years based on a combination of experimental reference data (X-ray structures) and quantum-mechanical computations. ${ }^{25}$ Experimental benchmark data is nonexistent for alloy surfaces and very limited for monometallic surfaces, which explains the slow development of metal/water force fields.

The motivation for developing a metal/water force field not only lies in gaining an atomistic understanding of the the metal/water interface, but also in being able to account adequately for the solvent effects, when investigating reactions at these interfaces. ${ }^{26-28}$ Solvent effects have, for instance, been shown to change the relative catalytic activity of metallic catalysts compared to the gas-phase ${ }^{29}$ and are key for a realistic description of electrocatalysis. ${ }^{30}$ Today, these investigations mostly rely on microsolvation ${ }^{29,31-35}$ or implicit solvents, ${ }^{36-39}$ where few water molecules or just a dielectric medium is used to represent the solvent, respectively. To overcome the limitations in sampling, molecular dynamics appears as a tool of choice. However, the use of DFT to perform MD simulations of relevant length $(\sim 500 \mathrm{ps})$ on systems with a relevant size $(\sim 200$ metal atoms and $\sim$ 200 water molecules) is computationally prohibitive for entire reaction pathways, ${ }^{23,40-42}$ since each ps of such a simulation requires $1^{\prime} 000-10^{\prime} 000$ CPUh. ${ }^{21}$

A hybrid approach, where the solvent is described at the MM and the surface at the DFT level, is a promising alternative, provided an accurate force field is available. ${ }^{43-46}$ To make such a strategy widely applicable, the metal/water force field should be compatible with established MM water models and standard Lennard-Jones and point-charge description of additional molecules and ions. This precludes the use of specialized force 
fields such as (AI)REBO, ${ }^{47-49} \mathrm{COMB}^{22}{ }^{22}$ ReaxFF, ${ }^{50,51}$ RexPON $^{52}$ or Neural Networks ${ }^{53}$ as the corresponding functional form is not compatible with well established force fields available for most organic molecules.

A weak chemisorption ${ }^{54}$ interaction of $5-10 \mathrm{kcal} / \mathrm{mol}$ drives the adsorption minimum of water molecules on the top position on closed-packed transition-metal surfaces. ${ }^{14}$ Chemisorption interactions are typically treated via fixed topologies in force fields. However, the metal/water interaction is weak and therefore needs to be formed and broken dynamically. For geometric reasons, an atom centered pair-wise potential yields an adsorption minimum in the hollow position as with the METAL ${ }^{15}$ or DFT-CES ${ }^{24}$ force field, in disagreement with the preferred top adsorption. Hence, non-conventional approaches are required to stabilize the top adsorption configuration compared to the hollow site. The use of projectors, ${ }^{11}$ reactive angular terms, ${ }^{12}$ virtual sites ${ }^{17}$ or explicit three body terms ${ }^{22}$ have been proposed to this effect. Our initial force field, GAL17, ${ }^{21}$ standing for Gaussian, Angular and Lennard-Jones, relies on attractive Gaussians which modulate the relative stability of top and hollow sites. In combination with the angular dependence terms and hydrogen repulsion, GAL17 retrieves the qualitative behavior of a single molecule adsorption on $\mathrm{Pt}(111)$ as a function of the distance, top/hollow position and angular orientation compared to DFT reference data. ${ }^{21}$ Furthermore, when coupled with an appropriate model for the water-water interaction, ice-like layers are well described as well.

This non-polarizable ${ }^{55}$ force field can be coupled to standard force fields and is compatible with any water-water interaction. However, GAL17 was developed having monometallic, perfectly flat single-crystal surfaces in mind. This allowed to simplify several terms (angular dependence, hydrogen repulsion) into expressions that only depend on the surface position. The extension of the force field from $\mathrm{Pt}(111)$ to other noble-metal surfaces also calls for a description of alloy surfaces. An energy expression such as GAL17 that depends on the global surface is not suitable to describe alloys, where the properties of the surface depends on the local configuration, i.e., in an $\mathrm{Ag} / \mathrm{Pd}$ alloy a locally Pd rich surface 
configuration will interact more strongly with water than an Ag rich configuration, in

analogy to other adsorbates. ${ }^{56-58}$ Hence, we here present a generalized energy expression, called GAL19, that is based on a pair-wise formulation in order to seamlessly treat alloy surfaces. GAL19 is made available in the free, open-source code CP2K.

The next section presents the details of the revamped GAL19 force field and the reference data to fit it for the (111) and (100) surface of $\mathrm{Cu}, \mathrm{Pd}, \mathrm{Ag}, \mathrm{Au}$ and Pt. After a short comparison of theses different surfaces for water adsorption, we discuss the quality of GAL19 for single molecule adsorption and then move to a comparison of the water structuration at the surface as a function of the metal and the facet. Finally, we investigate the interface of the $\mathrm{Ag} / \mathrm{Cu}$ and $\mathrm{Au} / \mathrm{Pt}$ alloys.

\section{Theory}

\subsection{Object definitions and functional form}

To define the adsorption of a single water molecule, several geometric descriptors can be distinguished: (i) the distance of the oxygen atom to the surface (ii) the relative position with respect to the surface atoms, e.g., the top, bridge and hollow position (see Fig. 1) and, (iii) the relative orientation of the $\mathrm{O}-\mathrm{H}$ bonds with respect to the surface normal (see Fig. $2 b)$.

In GAL19, all these definitions are required to be based on an atom pair-wise description. For instance, the distinction between top/hollow/bridge is well reproduced by sums over interatomic distances in combination with suitable functions to tune the relative interaction energies. The situation becomes more involved for the relative position of the $\mathrm{O}-\mathrm{H}$ bonds with respect to the surface normal. First, we have to define a surface normal $(\vec{n})$ based on interatomic pairs. In GAL19, we define a surface normal for each metallic 


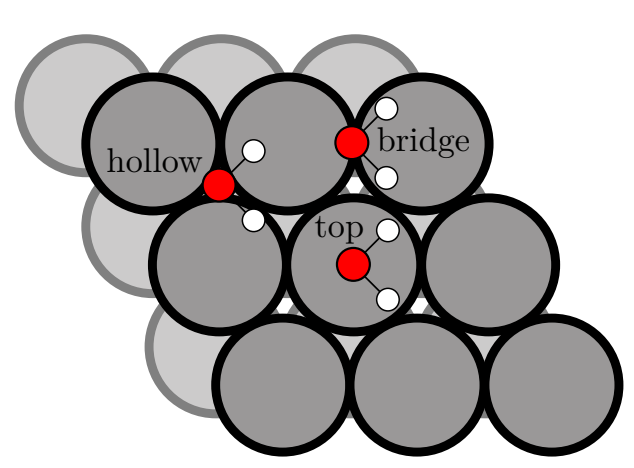

(111)

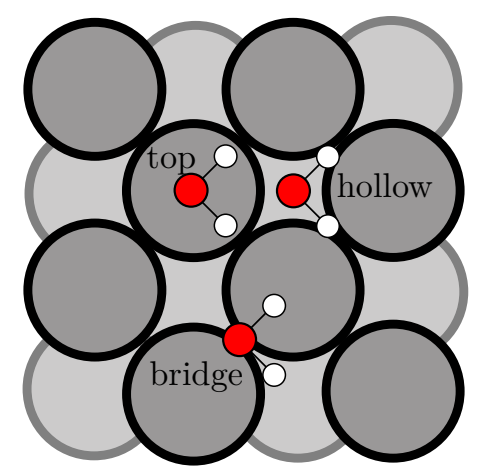

(100)

Figure 1: Schematic representation of the top, bridge and hollow site of adsorption on metallic (111) and (100) surfaces.

atom $M$ via:

$$
\vec{n}(M)=\sum_{i} \vec{r}_{M_{i}, M}
$$

where $i$ runs over all metallic atoms (i.e., including all constituents in the case of an alloy) within a distance cut-off of $M$ (see the green circle in Fig. 2a). Due to the symmetry of the surface and the underlying bulk, this vector always points perpendicular to the surface. In our implementation, the cutoff is set independently of the global force field cutoff. A value of $3.0 \AA$ has been used to include all first neighbors of the metallic atom. This is thus large enough to ensure a well behaved surface normal. Note that the very notion of a surface normal restrains the applicability of GAL19 to objects with locally well defined surfaces, i.e., neither to single atoms nor very amorphous or completely irregular nanoparticles with rough cavities etc.

Having defined $\vec{n}(M)$, the orientation of a water molecule is most conveniently expressed using three angles (see Fig. 2b): $\theta$, which is the angle between the dipole moment of the water molecule $\vec{\mu}$ and the surface normal, describes the cartwheel motion. $\phi$ is related to the propeller motion, defining the rotation of the water plane around the axis of the dipole moment. Finally, the helicopter angle $\omega$ describes the rotation of the water molecule around the surface normal.

In agreement with the pair-wise interaction potential, the general functional form of 
GAL19 reads:

$$
V_{\mathrm{GAL19}}=\sum_{H} \sum_{M \in \Omega(H)} \mathrm{V}_{M, H}\left(\vec{r}_{M, H}\right)+\sum_{O} \sum_{M \in \Omega(O)} \mathrm{V}_{M, O}\left(\vec{r}_{M, O}, \theta\right)
$$

where $\Omega$ represents an ensemble of metallic atoms (indistinct of their nature in the case of an alloy) within a given distance cut-off (red dotted circle in Fig. 2a). To simplify the notation below, we will not specify the nature of the metallic atom. In other words, it is implicit that when simulating alloys, the parameters for the corresponding $M, O$ or $M, H$ pair is used.

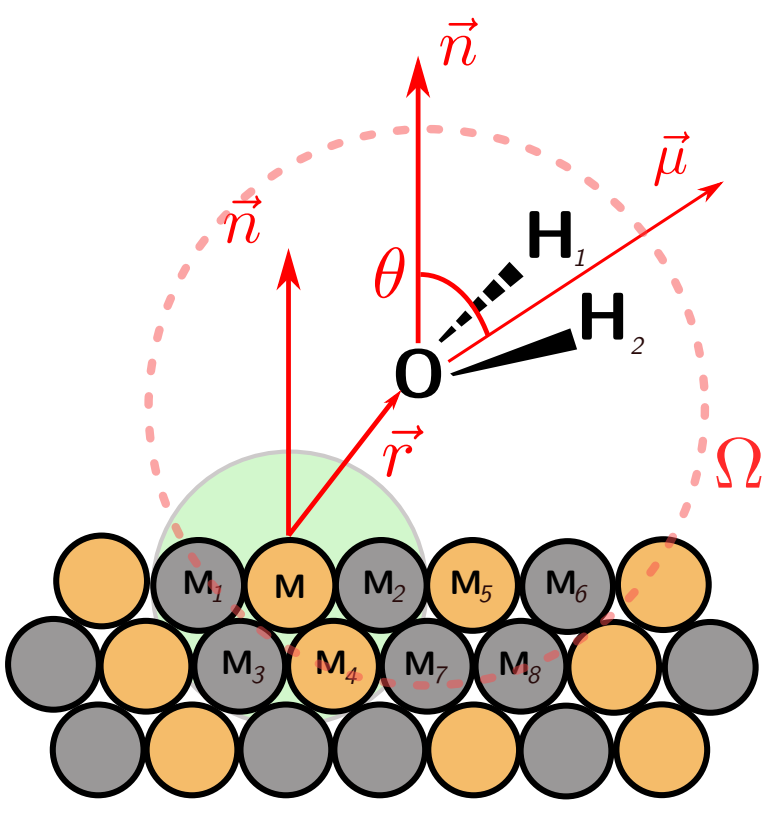

(a) Vectors for GAL19 definition.
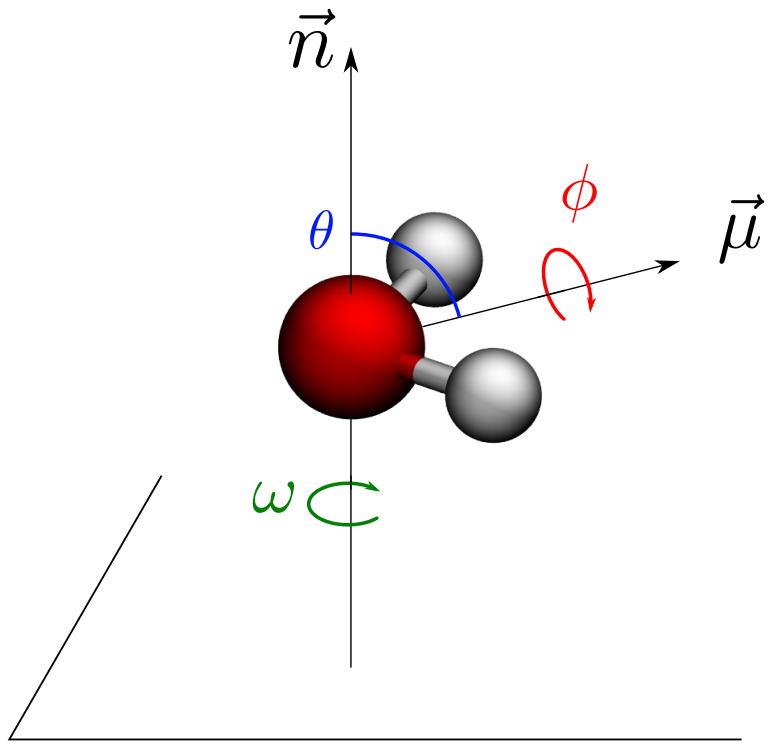

Figure 2: (a) Atoms, vectors and angle used in the definition of the GAL19 force-field for $\mathrm{M}-\mathrm{OH}_{2}: \vec{r}$ is the Metal/Oxygen vector; $\vec{n}$ is the surface normal defined by Eq. 1 for M using neighbouring metallic atoms within a cutoff distance shown by a green disk; $\vec{\mu}$ is the dipole of the water molecule; $\Omega$ is the ensemble of atoms within the cutoff (red dotted circle) which contribute to the overall interaction energy of the water molecule with the metallic surface; and $\theta$ is the angle between $\vec{n}$ and $\vec{\mu}$. (b) Schematic representation of the cartwheel angle $\theta$, the propeller angle $\phi$, and the helicopter angle $\omega$, defining the orientation of the water molecule with respect to the surface. $\omega=0$ is arbitrarily defined as a coincidence of the dipole moment vector and the x-axis. At $\phi=0$ the molecular plane of $\mathrm{H}_{2} \mathrm{O}$ is parallel to the surface.

As shown when developing GAL17, the helicopter angle $\omega$ is of negligible importance. 
In contrast, the propeller motion $\phi$ is key and is most conveniently described by the hydrogen repulsion with the metallic surface. In GAL19, this takes the the form of an exponential repulsive wall:

$$
\mathrm{V}_{\mathrm{M}, H}\left(r_{M, H}\right)=A_{H} e^{-r_{M, H} / R_{H}}
$$

were $r$ is the norm of $\vec{r}, A_{H}$ tunes the strength of the repulsion and $R_{H}$ is the characteristic distance of the exponential decay.

All the other interaction energy terms are collected in the metal oxygen pair:

$$
\mathrm{V}_{M, O}\left(\vec{r}_{M, O}, \theta\right)=\mathrm{V}_{G}\left(\varepsilon_{a}, b_{\|}, b_{\perp} ; \vec{r}_{M, O}\right)+\mathrm{V}_{A}\left(R_{O}, a_{1-4} ; r_{M, O}, \theta\right)+\mathrm{V}_{T T}\left(A, B, C_{6} ; r_{M, O}\right)
$$

where $\mathrm{V}_{T T}$ is the potential of Tang and Toennies, ${ }^{59} \mathrm{~V}_{G}$ is an attractive Gaussian and $\mathrm{V}_{A}$ is the term for the explicit $\theta$ angular dependence. Note that for a better readability, the dependence of these expressions on the surface normal and similar ensemble averages that include all metal atoms within the cutoff-distance $(M \in \Omega(O))$ are omitted here. These terms are explicitly described below. $A, B, C_{6}, \varepsilon_{a}, b_{\|}, b_{\perp}, R_{O}, a_{1}, a_{2}, a_{3}$, and $a_{4}$ are the respective parameters which are determined as described in section 2.2.

\subsubsection{Physisorption term: $\mathbf{V}_{T T}$}

The physisorption potential $\mathrm{V}_{T T}$ is taken from the seminal work of Tang and Toennies, ${ }^{59}$ truncated to the typical (London) dispersion expression $\frac{C}{r^{6}}$ :

$$
\mathrm{V}_{T T}(r)=A e^{-B \cdot r}-\left[1-\sum_{k=0}^{6} \frac{(B \cdot r)^{k}}{k !} e^{-B \cdot r}\right] \frac{C_{6}}{r^{6}}
$$

where $A, B$, and $C_{6}$ are parameters.

The Tang and Toennies potential is closely related to the Born-Mayer ${ }^{60}$ or Buckingham potential, ${ }^{61}$ with which it shares the exponential soft-wall potential and the long-range 
London attraction. However, in contrast to these earlier potentials, the one of Tang and Toennies damps the diverging $\frac{C_{6}}{r^{6}}$ potential in order to have a continously repulsive potential in the short range. The particularity of the Tang and Toennies damping function is that it does not rely on any additional parameters, but just re-uses the steepness parameter $B$ of the exponentially decaying repulsion. Note that the Tang and Toennies damping function is successfully exploited in dispersion corrections to $\mathrm{DFT}^{62}$ and in particular in $\mathrm{dDsC}^{63}$ which is the one used herein.

\subsubsection{Chemisorption terms: $V_{G}$ and $V_{A}$}

DFT computations and surface experiments ${ }^{64}$ agree that the top site is preferred for the adsorption of a water molecule. Hence, in order to stabilize the top site with respect to the hollow site, which would be the low-energy site when only using the physisorption potential (Eq. 5), we rely on the polarized attractive Gaussian potential introduced in the GAL17 force field: ${ }^{21}$

$$
\mathrm{V}_{G}\left(\vec{r}_{M, O}\right)=\varepsilon_{a} e^{-b_{\|} \cdot r_{\|}^{2}} e^{-b_{\perp} \cdot r_{\perp}^{2}}
$$

were $r_{\|}$and $r_{\perp}$ are, respectively, the parallel and perpendicular projection of $\vec{r}_{M, O}$ on the surface normal $\vec{n}$.

The asymmetry introduced by distinguishing the direction perpendicular and parallel to the plane allows to tune independently the contribution of the Gaussian on the top and hollow site (see Fig. 3). In a first approximation, the Gaussian of each metallic atom in an alloy surface is taken from the corresponding monometallic surface. To improve the approximation and include at least some fraction of the electronic effects present in alloys, one could introduce atom types that depend on the environment. These atom types would, themselves be parametrized via a cluster expansion, as we have previously done in the context of acetylene adsorption on $\mathrm{Ag} / \mathrm{Pd}$ alloys. ${ }^{58}$ The introduction of atom types avoids the use of many-body terms to introduce a dependence on the local environment. The explicit many-body terms are, in our case, computationally unnecessarily expensive, since 
the corresponding atoms do not move during a given computation.

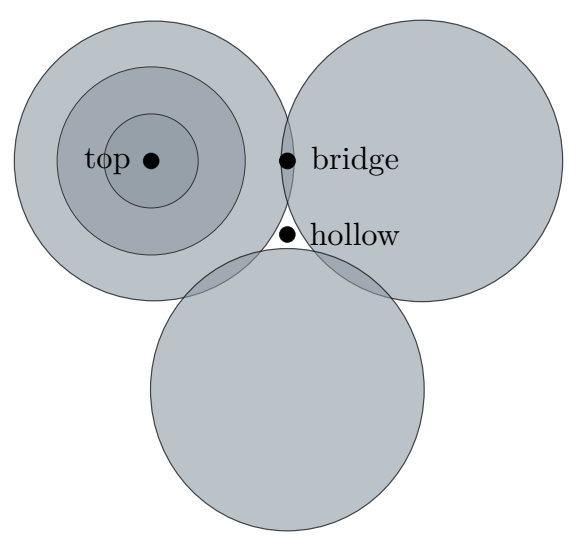

Figure 3: Schematic representation of attractive Gaussian potentials centered on the position of the nuclei. The Gaussians overlap more strongly on bridge sites than on hollow sites. Lines represent isopotential surfaces projected on the surface. Darker colors indicate stronger attractive potential. Metallic atoms are situated at the centre of the circles.

The second chemisorption term of GAL19 is an explicit angular dependence. Indeed, according to DFT, the angle $\theta$ of the chemisorption minimum is $\sim 90^{\circ}$. To reproduce this angular preference, we rely on a damped, truncated Fourier series of 4 terms:

$$
\mathrm{V}_{A}\left(M_{i} \in \Omega(O) ; r, \theta\right)=\frac{\left(e^{-r / R_{O}}\right)^{2}}{\sum_{M_{i} \in \Omega(O)} e^{-r_{M_{i}, O} / R_{O}}} \sum_{n=1}^{4} \mathrm{a}_{n} \cos (n \theta)
$$

The angular dependence has to vanish for molecules in solution. In GAL17, this was achieved by a global definition of the position of the surface. In GAL19, however, we require a fully pair-wise additive formulation of all terms. Here, we choose an exponential decay with a characteristic distance of $R_{O}$. Directly imposing a distance decay for each atom would lead to an unequal description for top, bridge and hollow sites, since they have one, two and three nearest metal neighbours respectively. Squaring the distance dependence and renormalizing it with the sum of contributions within the cutoff $\Omega(O)$ provides a more balanced description across the entire surface. 


\subsection{Fitting method and data set}

In total, 13 adjustable parameters are needed for the GAL19 forcefield for each metal: the three parameters for physisorption (Eq. 5) $A, B$ and $C_{6}$; the three parameters of the Gaussian (Eq. 6) $\varepsilon_{a}, b_{\|}$and $b_{\perp}$; the five parameters for the angular dependence (Eq. 7) $R_{O}, a_{1}, a_{2}, a_{3}$, and $a_{4}$; and the hydrogen repulsion parameters $A_{H}$ and $R_{H}$ of Eq. 3.

In order to fit these parameters, a set of 826 configurations is built for each metal. All configuration consist of a single water molecule adsorbed on a $\mathrm{p}(3 \times 3)$ metallic slab. The configurations probe various orientations, distances and adsorption sites (top, hollow etc.) of the water molecule. Only a restricted part of the total set (about 250 configurations) is used to fit the data, while the rest is used to validate the model. More details about the set can be found in the supporting information.

The $C_{6}$ parameter is the only one that is not fitted but directly extracted from the DFT computations via the use of the $\mathrm{dDsC}$ dispersion correction. ${ }^{65} \mathrm{~A}$ configurationnal average over the fitting set has been chosen for the final $C_{6}$ value. In analogy to the typically adopted united atom approach in water force fields, ${ }^{66}$ we use a single $C_{6}$ coefficient for the water molecule, i.e., the combination of the hydrogen and oxygen metal $C_{6}$ coefficients.

For the other parameters we distinguish the eight linear $\left(A, B, a_{1}, a_{2}, a_{3}, a_{4}, A_{H}, \varepsilon_{a}\right)$ from the five non-linear ones $\left(B, b_{\|}\right.$and $b_{\perp}, R_{O}$, and $\left.R_{H}\right)$. The non-linear parameters are optimized via a simplex optimizer, distributed via the SIESTA package. ${ }^{67}$ In each iteration of the simplex, the optimal solution of the eight linear parameters is obtained via a least square procedure. This process drastically accelerates the optimization of the parameters and is, furthermore, more robust to deal with linear dependencies within the parameter set.

The optimal parameters for the five metals and the two facets are provided in SI. 


\section{Computational details}

\subsection{DFT}

All DFT single-point evaluations have been carried out with VASP $5.4 .1,{ }^{68,69}$ using the PBE generalized gradient approximation functional ${ }^{70,71}$ with the $\mathrm{dDsC}$ dispersion correction $^{63,72}$ and an energy cutoff of $400 \mathrm{eV}$ for the expansion of the plane-wave basis. The electron-ion interactions are described by the PAW formalism. ${ }^{73,74}$ The interatomic distance of the bulk metals have been optimized and found to be 2.56, 2.78, 2.92, 2.81 and $2.94 \AA$ for $\mathrm{Cu}, \mathrm{Pd}, \mathrm{Ag}, \mathrm{Pt}$ and $\mathrm{Au}$, respectively. For all five metals, we have investigated the (100) and (111) facets. Series of 826 configurations of a single water molecule adsorbed on a $\mathrm{p}(3 \times 3)$ metallic unit cell with 4 metallic layers were built for each of the metal/facet couple. The slabs are separated by a vacuum of $20 \AA$ in order to minimize interactions between periodic images. The diverse set (see SI) explores the configurational space characterized by the four main descriptors: The adsorption site, the distance to the surface, the cartwheel angle $\theta$ and the propeller angle $\phi$ as defined in Fig. 2b. The Brillouin zone was sampled by a $\Gamma$-centered $3 \times 3 \times 1$ Monkhorst-Pack K-point grid. ${ }^{75}$ Idealized geometries (as cut from the bulks) were adopted for the metallic layers, while the water molecule was taken from a DFT optimization in gas phase (O-H: $0.98 \AA$ and a $\mathrm{H}-\mathrm{O}-\mathrm{H}$ angle of $\left.105.32^{\circ}\right)$. The impact of the water geometry on the interaction energy at the $\operatorname{Pt}(111)$ interface is depicted in Fig. $\mathrm{S} 1$ and found to be small $(0.55 \mathrm{kcal} / \mathrm{mol}$ per water molecule on average), so that it barely affects relative energies. Furthermore, choosing a geometry that is not taken from a specific MM water model makes GAL19 less bound to a given water model. Indeed, we combine GAL19 not only with TIP3P, but also perform tests with OPC $3^{76}$ and a polarizable water model. ${ }^{77}$ 


\subsection{Molecular Mechanics}

All molecular mechanics simulations have been carried out with CP2K 5.1, ${ }^{78-81}$ using the FIST module under periodic boundary conditions. Long-range Coulombic interactions were evaluated through the smooth particle-mesh Ewald summation. ${ }^{82}$ Molecular dynamics were run in the NVT ensemble at $300 \mathrm{~K}$, using the default settings for the NoseHoover thermostat. ${ }^{83,84}$ Water molecules interactions were simulated according to the TIP3P model ${ }^{66}$ and inter-atomic distances were accordingly constraint. All metallic atoms were kept frozen. The water/metal interactions were described by our implementation of the GAL19 force-field, which will be released to the public in a future version of the CP2K software.

The interfacial systems contained 192 metal atoms $(\mathrm{c}(4 \times 6), 4$ layers $)$ and 250 water

molecules, yielding a water layer of about $20 \AA$. A vacuum layer of $20 \AA$ separates the water from the "downside" of the metal slab, see Fig. S2. Since we do not apply a barostat, this avoids the simulation of a confined water layer. Finite size effects were tested by running simulations with a larger unit cell (432 metal atoms). As shown in Fig. S3, these simulations gave nearly identical results for $\mathrm{Pt}(111)$ as the smaller unit cell, suggesting a limited dependence on the box size beyond the one adopted herein. For the simulations of random alloys we used Vegard's law ${ }^{85}$ to determine the lattice constants, i.e., linear interpolations between the monometallics according to their molar fractions. The random distributions of the 192 atoms were generated such that each metallic layer has the target ratio between the two metals. Just like the monometallics, the alloy surfaces have been kept fixed in their idealized bulk geometries during the simulations. In all simulations, the interfaces were equilibrated for 400 ps and averages were then calculated over 600 ps, giving total simulation lengths of $1 \mathrm{~ns}$. 


\section{Results and discussion}

\subsection{Low-coverage water adsorption}

Energy Minima Water adsorption at 1/9 monolayer (ML) on the (111) and (100) facets of $\mathrm{Cu}, \mathrm{Pd}, \mathrm{Ag}, \mathrm{Pt}$ and $\mathrm{Au}$ has been studied by DFT and used for fitting GAL19 on these ten metallic surfaces.

Water adsorbing flat on top sites $\left(\theta \approx 90^{\circ}, \phi=0^{\circ}\right)$ is the most stable conformation adsorption according to previous DFT optimization and experimental observations. ${ }^{64}$ Therefore, Fig. 4 reports the distance dependence of this adsorption modes for the 10 facets at the PBE-dDsC level of theory, used herein to fit GAL19. This level of theory has been validated against experimental data on $\mathrm{Pt}(111)$ in a previous study. ${ }^{72}$ Furthermore, the comparison of the minimum adsorption energy on the (111) surface with other vdWinclusive functionals ${ }^{86}$ shows a good agreement, with deviations of about $0.5 \mathrm{kcal} / \mathrm{mol}$ and $0.05 \AA$ for minimum adsorption energies and distances.

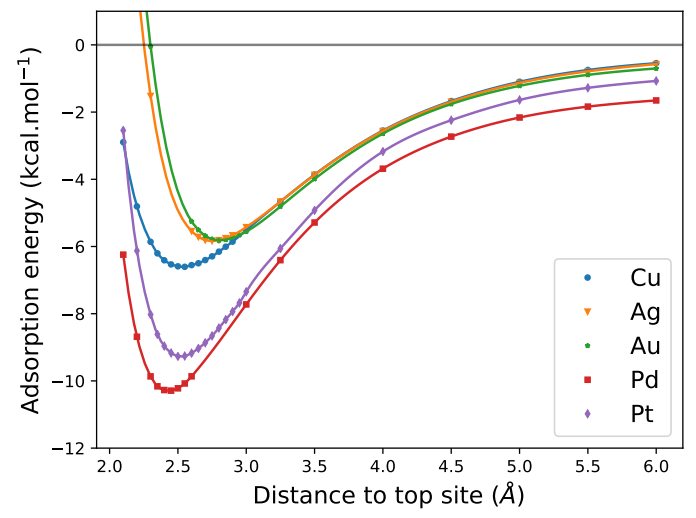

(a) 111 surfaces

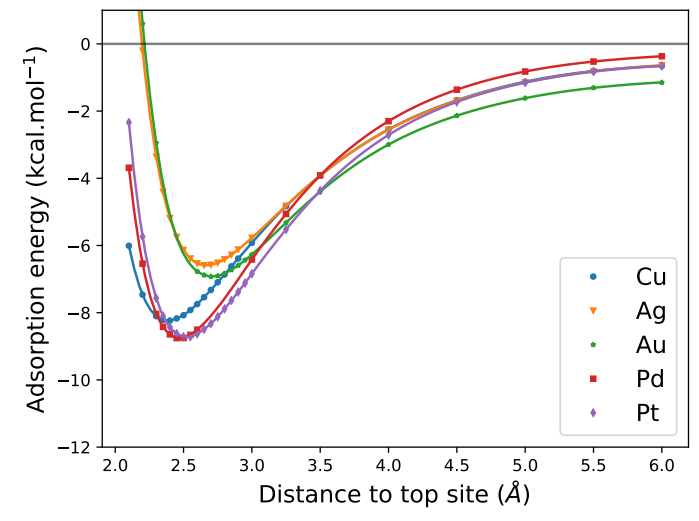

(b) 100 surfaces

Figure 4: DFT calculated adsorption energy of a water molecule on all the considered metals for their (111) facet (a), and (100) facet (b), in function of the distance of the water molecule's oxygen to a top site of the surface, with the angles $\theta$ and $\phi$ (as defined in Fig. 2 ) held at $90^{\circ}$ and $0^{\circ}$ respectively.

Starting with the (111) surfaces, which is the lowest energy facet of these fcc metals, Fig. 4a shows the (dis)similarities of the studied metals. This analysis agrees well with earlier 
reports, 87,88 but sets the stage for the subsequent discussion. Water binds only weakly (5.8 $\mathrm{kcal} / \mathrm{mol})$ to $\mathrm{Au}(111)$ and $\mathrm{Ag}(111)$ surfaces at equilibrium distances of 2.8 and 2.75 $\AA$ respectively. The third coinage metal, $\mathrm{Cu}(111)$, binds water slightly more strongly (6.6 $\mathrm{kcal} / \mathrm{mol})$ at a shorter distance $(2.55 \AA)$, in agreement with its smaller size, reflected in the smaller lattice constant (2.56 ̊ interatomic distance vs. $2.94 \AA$ for $\mathrm{Au})$. Finally, $\operatorname{Pt}(111)$ and $\operatorname{Pd}(111)$ bind water the most strongly at the shortest distance (2.5 and $2.45 \AA$ respectively), despite their large lattice constant $(\approx 2.8 \AA$ interatomic distance). This can be rationalized by the significant chemisorption contribution of water on these surfaces. ${ }^{54}$

Moving to the (100) surfaces (Fig. 4b), which are the second most stable ones for these metals, the overall ordering with respect to the energy minimum remains the same as on the (111) facet. However, the coinage metals adsorb water more strongly on the (100) than on the (111) surface, which is most noticeable for $\mathrm{Cu}(100)(8.23 \mathrm{vs} 6.6 \mathrm{kcal} / \mathrm{mol})$, while it is the other way round for Pd and Pt. For more strongly adsorbed species such as methyl $\left(\mathrm{CH}_{3}\right)$ or $\mathrm{CO}$, a "universal" relationship has been identified, ${ }^{89}$ according to which the more compact facets are interacting less strongly with adsorbates than more open facets due to the higher degree of unsaturation of metallic bonds in the latter. Apparently, this "universal" observation does not necessarily hold for water on (111) vs. (100) and could, therefore, impact the relative stability of the two facets in water compared to computations in vacuum. The preferred shape of large nanoparticles in water compared to vacuum can be determined using Wulff-reconstructions. ${ }^{90}$ Nevertheless, determining the surface free energies of aqueous (100) and (111) surfaces is beyond the scope of this paper: extensive tests with our previous version of the force field, GAL17, have shown that these simulations are technically challenging and we will report on the corresponding results elsewhere. Furthermore, the relative energies of small nanoparticles require also an energy expression for the metal-metal interactions, which is beyond the capabilities of GAL19. For the same reason, surface reconstructions in water cannot be properly described. Overall, we conclude that $\mathrm{Pt}$ and $\mathrm{Pd}$ surfaces together with $\mathrm{Cu}(100)$ bind water strongly, while 
$\mathrm{Cu}(111), \mathrm{Au}$ and $\mathrm{Ag}$ are significantly less oxophilic.

Fit quality of GAL19 A parity plot of GAL19 compared to the DFT reference data is shown for $\mathrm{Au}(100)$ and $\mathrm{Pd}(111)$ in Fig. 5. These two surfaces are typical for a weak and a strong binding of $\mathrm{H}_{2} \mathrm{O}$ and the corresponding graphs for the other surfaces are available in the SI. The root mean square deviation of the error of GAL19 (see Table S3 in the SI) ranges from 0.8 (for $\mathrm{Cu}(100))$ to $1.4 \mathrm{kcal} \cdot \mathrm{mol}^{-1}(\mathrm{Pt}(100))$ with an average of $1.0 \mathrm{kcal} \cdot \mathrm{mol}^{-1}$. This demonstrates the very satisfying overall performance of GAL19. To test the importance of the training set, we have performed a second parametrization with a randomized training set for $\mathrm{Au}(111)$ and $\mathrm{Pt}(100)$, which have low $(0.75 \mathrm{kcal} / \mathrm{mol})$ and high $(1.39 \mathrm{kcal} / \mathrm{mol})$ RMSDs, respectively. The randomized fit set yields, with 0.98 and $1.19 \mathrm{kcal} / \mathrm{mol}$ very similar RMSDs. This similarity is also reflected in the parameters (see Table S2). This demonstrates that the functional form and optimization method are robust.

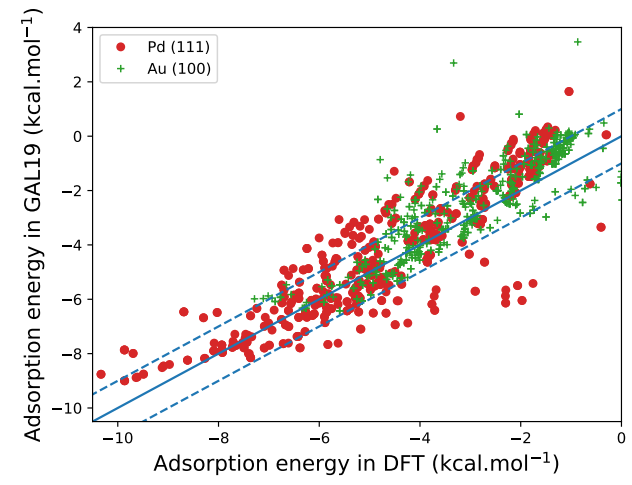

Figure 5: Comparison of the adsorption energy of multiple adsorption conformations of a single water molecule on Pd (111) and Au (100), calculated by DFT and GAL19. All the configurations of the total set (see Supplementary information), resulting in an adsorption energy lower than $0 \mathrm{kcal} / \mathrm{mol}$ in DFT are included. The broken lines indicates errors of \pm $1 \mathrm{kcal} / \mathrm{mol}$.

To analyze the performance of GAL19 more specifically, we first verify the angular dependence, which we previously identified to be a critical point of comparison between different force fields. ${ }^{21}$ Fig. 6 shows the $\theta$ dependence of the adsorption energy on $\operatorname{Pt}(111)$ which is representative of all surfaces. The $\phi$ dependence and the corresponding graphs 
for the other surfaces are shown in the SI. Overall, the $\theta$ dependence shows the success of GAL19 to reproduce the DFT reference data faithfully and in particular the minimum around $90^{\circ}$ is reproduced in contrast with the previous generation, where the minimum was shifted to $60^{\circ}$.

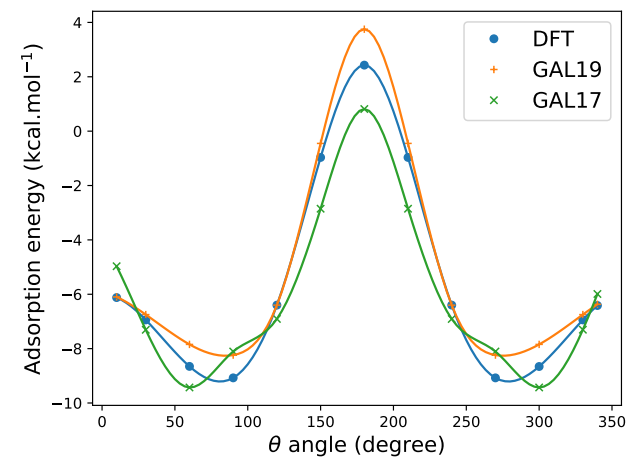

Figure 6: DFT, GAL17 and GAL19 calculated adsorption energy of a water molecule on Pt (111) in function of the cartwheel angle $\theta$, with $\phi$ held at $0^{\circ}$ and at $2.5 \AA$ from a top site.

The DFT energy minima discussed above are well reproduced by GAL19 (see Fig. 7 and Table 1 and the additional data in the SI). Despite GAL19 being designed to reproduce the chemisorption minimum, Fig. 7 evidences the largest errors, up to $1.3 \mathrm{kcal} / \mathrm{mol}$ for $\mathrm{Pd}(111)$ for the more oxophilic surfaces. Nevertheless, overall the trends are well reproduced, both between metals but also between facets, see Fig. S4 and S5. The biggest deviation for the difference between (100) and (111) is obtained for $\mathrm{Cu}$, where it amounts to $0.8 \mathrm{kcal} / \mathrm{mol}$ in GAL19, while it should be $1.6 \mathrm{kcal} / \mathrm{mol}$ according to DFT.

The positions of the top and hollow minima are also compared between DFT and GAL19. The position of the minimum adsorption energy on top and hollow site $\left(r_{\text {min,top }}\right.$ and $r_{\text {min,hlw }}$, respectively) and their relative stability $\left(\Delta E_{t o p, h l w}\right)$ are available in the SI and the deviations $\left(\Delta_{D F T, G A L 19}\right)$ reported in Table 1 . The low average errors $(\sim 0.1 \AA$, and $0.5 \mathrm{kcal} / \mathrm{mol}$ ) demonstrates the capacity of GAL19 to describe the chemisorption that is different between the hollow and the top site according to DFT. This relative stability of the top site is governed by the anisotropic Gaussian (Eq. 6), which counterbalances the 


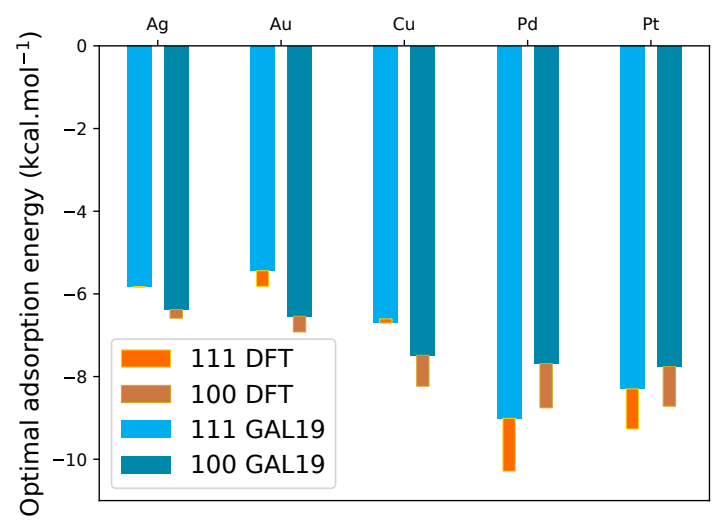

Figure 7: DFT and GAL19 calculated adsorption energy of a single water molecule on different metallic facets, corresponding to the minimum energy found by varying the adsorption distance on top site, with $\theta$ and $\phi$ (as defined above) held at $90^{\circ}$ and $0^{\circ}$ respectively.

physisorption term (Eq. 5). The physisorption alone yields an energy minimum for the hollow site, as observed for the METAL force field ${ }^{15}$ and the DFT-CES. ${ }^{24}$ Reproducing the adsorption energy and position away from the top site (e.g., on hollow) is crucial when aiming at a realistic description of the metal/water interface. If there is no strong preference of water molecules for the top site, one might, for instance, expect a much more disordered interface than when molecules are almost immobilized on the top site.

Table 1: Maximum and average deviation of the location of the minimum between DFT and GAL19 $\left(\delta r_{\text {min }}\right)$ for top and hollow (hlw) site adsorption and of $\delta \Delta E_{t o p} / h l w . \delta r_{\text {min }}$ is defined as the differences between optimal adsorption distance from the surface in DFT and GAL19 for a single water molecule on the given site and with with $\theta$ and $\phi$ at $90^{\circ}$ and $0^{\circ}$ respectively. Likewise, $\delta \Delta E_{\text {top } / h l w}$ is defined by $\delta \Delta E_{\text {top } / h l w}=\Delta_{G A L 19} E_{\text {top } / h l w}-$ $\Delta_{D F T} E_{t o p / h l w}=\left(E_{\text {min }, h l w, G A L 19}-E_{\text {min }, \text { top }, G A L 19}\right)-\left(E_{\text {min }, h l w, D F T}-E_{\text {min }, \text { top }, D F T}\right)$ where $E_{\text {min }}$ corresponds to the energy minimum.

\begin{tabular}{ccccc}
\hline & Maximum & Average & Max\% & Average\% \\
\hline$\delta r_{\text {min,top }}(\AA)$ & 0.2 & 0.095 & 8.0 & 3.7 \\
$\delta r_{\text {min, hlw }}(\AA)$ & -0.25 & -0.155 & 9.3 & 5.5 \\
$\delta \Delta E_{\text {top } / \text { hlw }}\left(\mathrm{kcal} \cdot \mathrm{mol}^{-1}\right)$ & 1.5 & 0.4 & 87 & 32 \\
\hline
\end{tabular}




\subsection{Ice layers on the (111) facets}

In contrast to liquid water/metal interface, the ice water monolayer/metal interface is quite extensively studied. ${ }^{91}$ In particular, the hexagonal water layer put forward by Doering et al. ${ }^{92}$ has been the cornerstone for the understanding of ice monolayers on noble metal surfaces. As a partial validation for more complex adsorption geometries, we have assessed the interaction energy of three typical ice-like layers over the (111) facets over the five metal surfaces. The most stable $\sqrt{3} \times \sqrt{3}$ unit cell, called $\mathrm{H}_{\mathrm{down}}$, is a typical honeycomb ice-like layer based on the work by Doering et al. ${ }^{92}$ Additionally, we also investigate less regular but more stable $\sqrt{37} \times \sqrt{37}$ and $\sqrt{39} \times \sqrt{39}$ unit cells that have been observed over $\operatorname{Pt}(111) .{ }^{93}$

The performance of GAL19 for these ice-like layers depends significantly on the metal studied (see Fig. 8). The slope of the GAL19 interaction energy vs. the DFT interaction energy is worst for $\mathrm{Cu}$ (almost flat), while for $\mathrm{Ag}$ and $\mathrm{Pd}$ the results are more acceptable (slope of 0.16 and 0.20 ). The $\mathrm{H}_{\text {down }}$ layer, where the water molecules are mostly on top sites, is the one that has the lowest interaction energy, but is also closest to the DFT reference values. For the larger and more complex structures $(\sqrt{37}$ and $\sqrt{39})$ where the water molecules are not situated at high-symmetry positions, the agreement is worse and, moreover, the relative stability from one metal to the other is completely washed out. The two origins for this discrepancy can be found in (i) the uneven description of the top/hollow energetic preference from one metal to the other and (ii) the absence of many-body water-water-metal interactions at the interface, which are non-negligible, ${ }^{94}$ but beyond the scope of the current force field. The good news is, however, that (a) there is a qualitative agreement of which adsorption layer is more stable than the other and (b) the energy difference between them is smaller than at the DFT level. This means that sampling at the MM level and re-evaluating the energy of snapshots at the QM level in a resampling spirit is likely to be successful as the MM energy landscape is flat enough to allow a significant ensemble overlap between $\mathrm{QM}$ and $\mathrm{MM}$ at room temperature. 


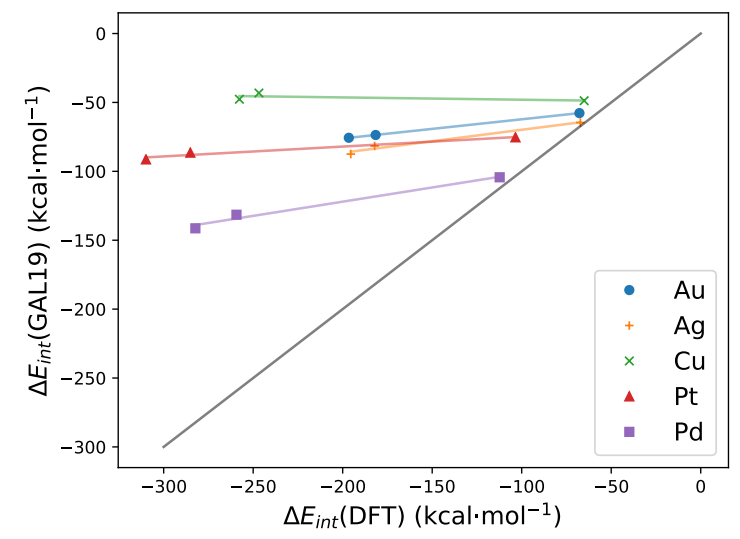

Figure 8: Interaction energies of GAL19 vs DFT for ice-like layers on the (111) facets.

\subsection{The mono-metallic/water interface}

Molecular dynamics simulations of $1 \mathrm{~ns}$ were carried out with GAL19 to evaluate the structure of the metal/water interface for $\mathrm{Pt}, \mathrm{Pd}, \mathrm{Au}, \mathrm{Ag}, \mathrm{Cu}$. Due to the slow diffusion of water at the interface, such an extensive phase-space sampling is necessary for obtaining equilibrated results. ${ }^{18,21}$ Fig. S6 reports the results of a 5 ns trajectory for $\operatorname{Pt}(111)$, which shows that $1 \mathrm{~ns}$ simulations are well converged. Experimentally, the evidence on the neutral metal/water interface is very scarce: Toney et al. have shown that the density in the first layer over $\operatorname{Ag}(111)$ is at least $30 \%$ higher compared to the bulk and that two to three layers can be distinguished. ${ }^{8}$ The IR data on the $\mathrm{Au}(111)$ and $\operatorname{Pt}(111)$ is debated ${ }^{95}$ but tends to suggest either flat ${ }^{96}$ or locally ice-like arrangements ${ }^{97}$ for the first layer. Most recent studies heavily rely on theoretical models ${ }^{9,10}$ to interpret the experimental data, but tend not to test many possibilities. As a result, the early ultra-high vacuum data for a water layer on ruthenium ${ }^{92}$ is still frequently used as a reference point to advocate "bilayers" with a $\sqrt{3} \times \sqrt{3}$ unit cell, even though more recent works have shown that the most stable monolayer requires a significantly larger $\sqrt{39} \times \sqrt{39}$ unit cell at least on $\operatorname{Pt}(111) .{ }^{93}$ Overall, as a result of the difficulties of the experiments, the (dis-)similarities between noble metal/water interfaces remain largely unaddressed by experiments.

The structuring of the interface can be characterized through the monitoring of various 


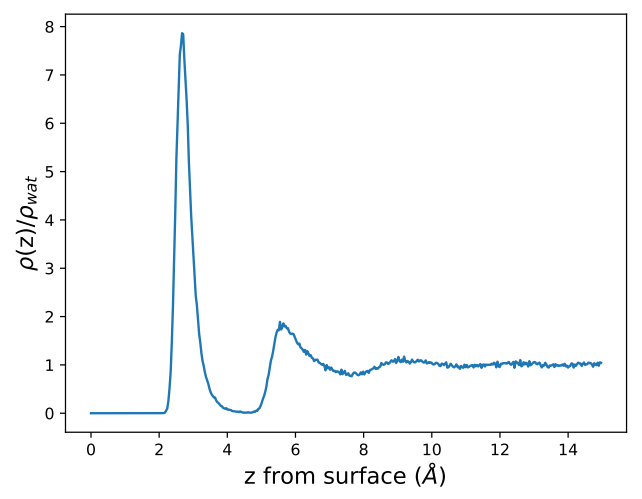

(a) $\rho(z)$ in terms of oxygen atoms.

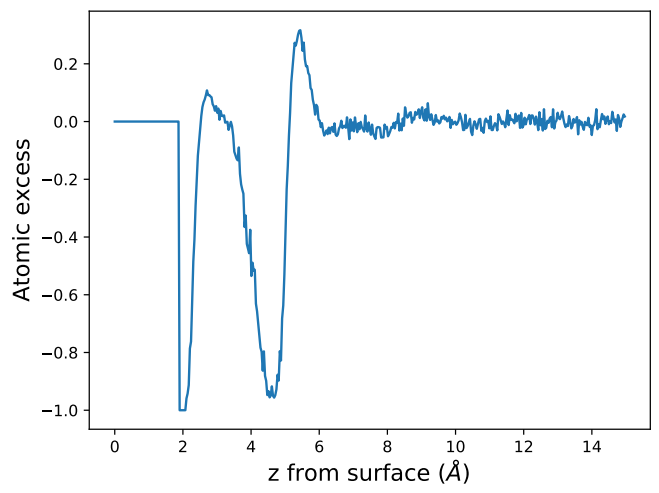

(b) Atomic excess.

Figure 9: Interface structuring as a function of the distance with respect to the metal surface (z) revealed by (a) the molecular density $\rho(z)$ divided by the reference density of water $\rho_{\text {wat }}$ and (b) the atomic excess $\mathrm{AE}(\mathrm{z})$ for the $\mathrm{Pt}(111) / \mathrm{H}_{2} \mathrm{O}$ interface. AE is held at 0 when neither oxygen or hydrogen are found in the layer. Layers of a thickness of $0.033 \AA$ are used.

averaged quantities. The planar average density, $d(z)=\frac{\rho(z)}{\rho(w a t)}=\frac{n_{O}(z-d z / 2, z+d z / 2)}{\Delta X \cdot \Delta Y \cdot \delta z * \rho(w a t)}$, as a function of the distance from the topmost metal nuclei (see Fig. 9a), is based on the position of the oxygen atoms and compare the number of oxygen atom $\left(n_{O}\right)$ found in each layer of small height $(\delta z)$ - cut from the complete simulation cell (of volume $\Delta X \cdot \Delta Y \cdot \Delta Z$ ) - to the standard concentration of aqueous water $\left(\rho(w a t)=0.33 \AA^{-3}\right)$. The "atomic excess" (Fig. $9 b), A E(z)=\frac{n_{O}(z)-2 n_{H}(z)}{n_{O}(z)+2 n_{H}(z)}$, compares the average count of hydrogens $\left(n_{H}\right)$ and oxygens $\left(n_{O}\right)$ in layers parallel to the surface to identify oxygen rich $(\mathrm{AE}>0)$ and oxygen poor $(\mathrm{AE}<0)$ regions. $A E(z)$ and $\rho(z)$ give complementary information on the layering of the solid/water interface. ${ }^{27}$ Typical results for this analysis are shown in Fig. 9 for $\mathrm{Pt}(111)$. As shown in SI (see Fig. S7 and S8), all the interfaces feature a very similar structuring, with two distinct layers below $13 \AA$. Even when using the parametrization from the randomized fit sets, the results remain very comparable (see Fig. S9).

In order to assess the influence of the adopted water model on the interfacial structuring, we have performed additional simulations with the polarizable water force field from Dang and Chang ${ }^{77}$ for the $\mathrm{Pt}(111) /$ water interface. While qualitatively the interfacial 
structuring is similar to the one obtained with the TIP3P model, the polarizable water model leads to a somewhat longer-ranged structuring: a weakly structured third layer with a flat peak at $\sim 9 \AA$ above the surface in the $\rho(z)$ profile (see Fig. S10) can be distinguished. Interestingly, the OPC3 water model, which is characterized by stronger water-water interactions compared to TIP3P, yields very similar results compared to the polarizable water model (see Fig. S11).

To gain further insight, we have also analyzed the angular distribution within the two identified layers and the bulk layer. Chosen in the interval 13-15.5 $\AA$ above the surface, this bulk layer shows the expected random distribution, demonstrating that it is wellpositioned and thick enough to be neither affected by the structuring of the metal/water interface, nor by the water/gas interface. Since also the angular distributions change only subtly between facets and metals, Fig. 10 shows the data of the $\operatorname{Pt}(111)$ surface, while the corresponding data is available in the SI (Fig. S12 and S13) for the other facets. Remarkably, the water organization was suggested to be different on $\mathrm{Pt}(111)$ and $\mathrm{Pt}(100)$ based on the Siepmann-Sprik water/Pt interaction potential. ${ }^{18}$ In contrast, GAL19 that uses a facet specific parameterization does not yield a significant difference for interfacial organization between the (100) and the (111) surface.

The first water layer (ending at $\sim 4.5 \AA$ ) is very dense (about twice as dense as bulk water), in qualitative agreement with the experimental data of Toney et al. ${ }^{8}$ This first water layer has a strong preference for $\theta \approx 90^{\circ}$ as illustrated in Fig. 10a. Furthermore, the propeller angle $\phi$ is close to $0^{\circ}$, which means that in the first layer the molecular plane of water is essentially parallel to the metallic surface in agreement with the experimental data by Ataka et al. ${ }^{96}$ This is also in line with the very small positive AE peak at $\sim 3 \AA$, characteristic for compensated hydrogen and oxygen densities. This oxygen rich region is sandwiched between hydrogen rich $(\mathrm{AE}<0)$ zones. The first one $(\sim 2 \AA)$ can be explained by the lower repulsion of hydrogen compared to oxygen, which allows some hydrogen atoms to approach closer to the surface than oxygen. The second one $(\sim 4.5 \AA)$ is composed 
of few hydrogen atoms pointing towards the second layer (since most water molecules lay flat according to the $\theta$ distribution) and mostly of hydrogen atoms pointing from the second layer to the first layer to maximize the hydrogen bonding, as we will detail in the next paragraph. The first layer might, thus, appear hydrophobic, as previously suggested by Chandler and co-workers based on simulations with the Siepmann-Sprik force field. ${ }^{18}$ Note, however, that this conclusion does not hold for the simulations with a polarizable water-water force field, which features a shoulder for $\mathrm{H}$-up configurations $\left(\theta \approx 60^{\circ}\right.$, see Fig. S10). This emphasizes the need for a future validation of the interfacial structuring based on $\mathrm{QM} / \mathrm{MM}$ resampling techniques.

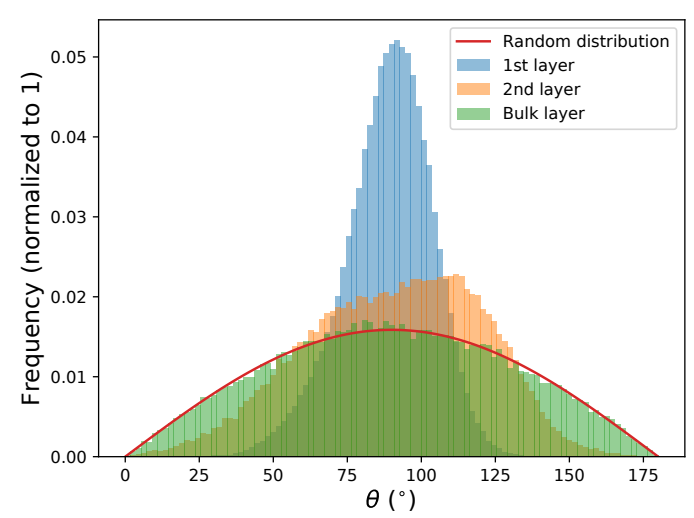

(a) $\theta$ Distribution

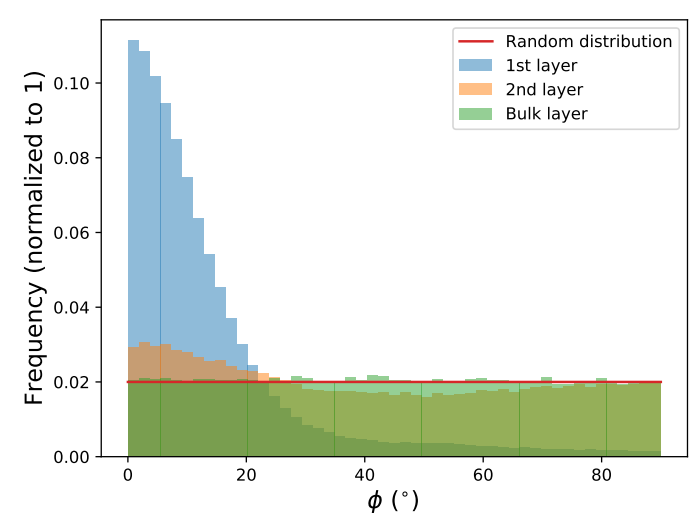

(b) $\phi$ Distribution

Figure 10: Angular distribution of the (a) the cartwheel angle $\theta$ and (b) the propeller angle $\phi$ of the water molecules on $\mathrm{Pt}(111)$ for chemisorption, physisorption, and bulk layer, defined as being water molecule situated between 0 and 4.5, 4.5 and 7, and 13 and $15.5 \AA$ away from the surface respectively. The red line represent an idealized random distribution of the dipoles.

The second water layer, which is still well distinguishable from the bulk, extends to $\sim 7$ $\AA$. It is only slightly denser than the bulk and has a much weaker orientational preference. The positive peak of $\mathrm{AE}$ at $6 \AA$, followed by a constant $\mathrm{AE} \approx 0$, and the peak at $\theta \approx 120^{\circ}$ both indicate that water molecules in this second layer are preferentially oriented "H-down" to interact with the first layer, the corresponding $\mathrm{H}$ atoms being in excess relative to the oxygen in the $\mathrm{H}$ rich region at $(\sim 4.5 \AA)$. A small population of the second layer is also 
oriented "H-up" (shoulder at $\theta \approx 60^{\circ}$ ). As shown in Fig. S12 and discussed below in the context of the $\mathrm{Pt} / \mathrm{Au}$ alloy, such a shoulder is already visible in the first layer for $\mathrm{Au}(111)$. The weak orientational preference of this second layer results from the balance between the water/water interaction and the metal/second layer water interaction since the explicit angular dependence (Eq. 7) drops to about $40 \%$ with respect to its value in the first layer. In summary, the first layer acts as a soft template for the second layer.

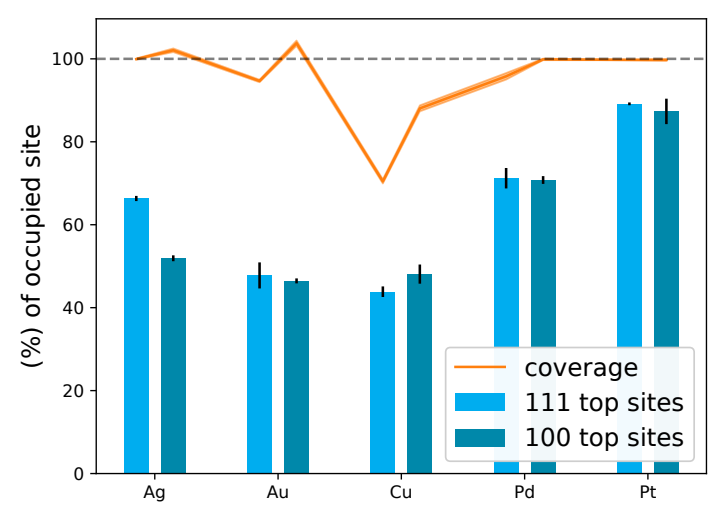

Figure 11: Percentage of the top sites occupied by water molecule and global coverage of the surface for multiple metallic facets. The coverage is defined as the number of oxygen atom in the first layer (between 0 and $4.5 \AA$ from the surface), divided by the number of surface atoms. Errors estimation at $95 \%$ confidence interval are depicted by enlarged line for total coverage and black bars for top site occupation.

Since the interface structuring is very similar for all ten surfaces studied (see Fig. S14 and S15), Fig. 11 compares the first adsorption layer in terms of coverage and adsorption site preference. The overall coverage (number of water molecules in the first layer divided by the number of surface atoms) reaches $100 \%$ for all surfaces but $\mathrm{Cu}(111)$. This is higher than the coverage for the famous bilayer structures $(67 \%),{ }^{92}$ but compatible with the high density of water the the $\mathrm{Ag}(111) /$ water interface measured experimentally. ${ }^{8}$ The lower overall coverage on $\mathrm{Cu}(111)$ can be traced back to the competition between adsorption (6.6 $\mathrm{kcal} / \mathrm{mol}$ in the minimum) and hydrogen bonding $(\sim 5 \mathrm{kcal} / \mathrm{mol})$ which is particularly fierce on $\mathrm{Cu}(111)$ since the $\mathrm{Cu}-\mathrm{Cu}$ distance of $2.56 \AA$ is too short to accommodate a hydrogen bond $(\mathrm{O}-\mathrm{H} \cdots \mathrm{O}$ of $\sim 2.9 \AA)$. However, when determining the proportion of 
water molecules on top sites (i.e., within $0.4 \AA$ of the formal top site), clear differences emerge between the metal surfaces. On Pt surfaces nearly all the top sites are occupied by water molecules. For Pd, the percentage drops to about $75 \%$, which can be explained by its top/hollow preference being at least $1 \mathrm{kcal} / \mathrm{mol}$ lower than for Pt. Because of the weaker binding on $\mathrm{Ag}$ and $\mathrm{Au}$, only $50-60 \%$ of top sites are occupied on these surfaces, clearly demonstrating more disordered interfaces. Such a comparative disordering has already been observed within the short ( $\sim 10$ ps) DFT based molecular dynamics simulations of Gross and co-workers. ${ }^{16}$ Since the lack of equilibration in the DFT-based MDs and the missing many-body contributions in our GAL19 force field make it difficult to a priori judge the relevance of the obtained results, the obtained agreement is reassuring for both approaches. The extent of the preference for top adsorption is expected to manifest when studying the adsorption of molecules on these surfaces, as disordered interfaces can accommodate adsorbates more easily than highly organized ones.

\subsection{Water structure at the alloy/water interface}

GAL19 enables investigations of alloy surfaces at the molecular mechanics level on the basis of accurate water adsorption and orientational preferences on monometallic surfaces. Fig. S16 and S17 show that GAL19 is capable of retrieving the major effects of alloying, even though electronic effects cannot be captured for obvious reasons. In the following, we study two families of random alloys to probe two distinct effects: First, the effect of alloying $\mathrm{Ag}$ with $\mathrm{Cu}$ is assessed. $\mathrm{Ag} / \mathrm{Cu}$ is a typical example of two surfaces with similar adsorption properties for a single water molecule, but a significant difference in lattice constant, leading to an interatomic distance of $2.56 \AA$ for $\mathrm{Cu}$ but $2.92 \AA$ for $\mathrm{Ag}$. Experimentally, $\mathrm{Ag} / \mathrm{Cu}$, which forms (metastable) solid solutions, ${ }^{98,99}$ is scrutinized for its anti-bacterial activity ${ }^{100}$ and is $\mathrm{CO}_{2}$ electroreduction properties. ${ }^{101}$ Second, $\mathrm{Au} / \mathrm{Pt}$ alloy surfaces, typical for nanoparticles with enhanced catalytic properties, ${ }^{103-105}$ are simulated to investigate the impact of mixing a strongly adsorbing metal $(\mathrm{Pt})$ with a 


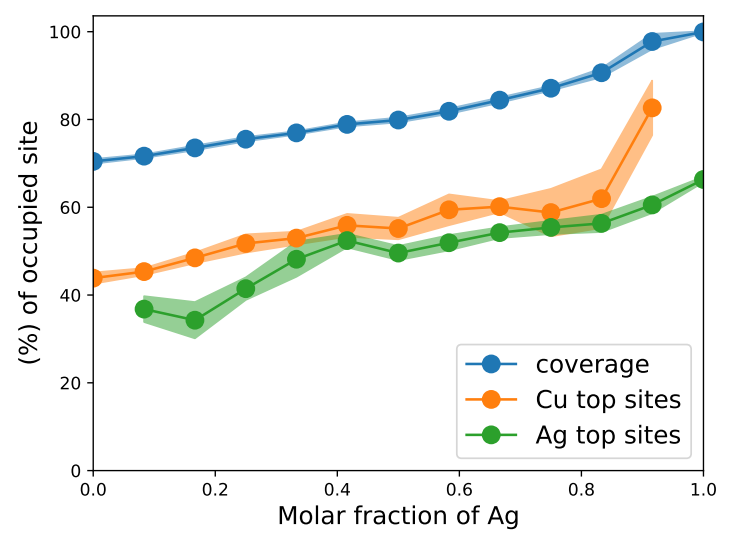

Figure 12: Percentage of the top sites of copper and silver occupied by water molecule and global coverage of the surface for multiple alloy composition of $\mathrm{Cu} / \mathrm{Ag}$. The coverage is defined as the number of oxygen atom in the first layer (between 0 and $4.5 \AA$ from the surface), divided by the total number of surface atoms. Confidence interval at $95 \%$ are represented as colored band surrounding the data. The corresponding variances were computed by block averaging. ${ }^{102}$

weakly interacting one $(\mathrm{Au})$ that have nearly the same lattice constant (2.81 and $2.94 \AA$ interatomic distance, respectively). As above, we monitor the total coverage and the percentage of occupied top sites to compare the different alloys. Furthermore, for the $\mathrm{Au} / \mathrm{Pt}$ alloy we also report the angular distributions.

The resulting curves for the $\mathrm{Cu} / \mathrm{Ag}$ (111) alloy are shown in Fig. 12. As expected, the percentage of occupied top sites is quite similar for both constituents, especially around an equimolar mixture. As shown in Fig. S18, this characteristic still holds when switching from the TIP3P water model to OPC $3,{ }^{76}$ which was found to behave differently at the interface in our previous study. ${ }^{21}$ At large Ag molar fractions, the remaining copper sites show an increased propensity to be occupied, which can be rationalized by the combined effect of a relatively stronger adsorption of water on copper than on silver (see Fig. 4a) and the increased lattice constant, which lifts the constraints on the hydrogen bond between adsorbed molecules. Indeed, the numerical experiment where the $\mathrm{Cu}(111)$ GAL19 parameters are used for a surface with the Ag lattice constant gives a high percentage of occupied top sites (95\%). The effect of the continuously increasing lattice constant is also 
seen in the smooth and almost linear increase in the overall coverage of the first water layer as the silver content increases. This increase could be experimentally verified adapting the techniques used for $\mathrm{Ag}(111)$ surfaces. ${ }^{8}$ Furthermore, the varying total coverage is expected to noticeably impact the solvation energies of adsorbates as a function of the alloy composition. In conclusion, the $\mathrm{Ag} / \mathrm{Cu}(111)$ alloy interface is expected to be disordered in analogy to the pure coinage metal surfaces (see Fig. 13). However, water preferentially adsorbs on copper sites, so that the silver atoms are more available to interact with other adsorbates compared to pure silver.
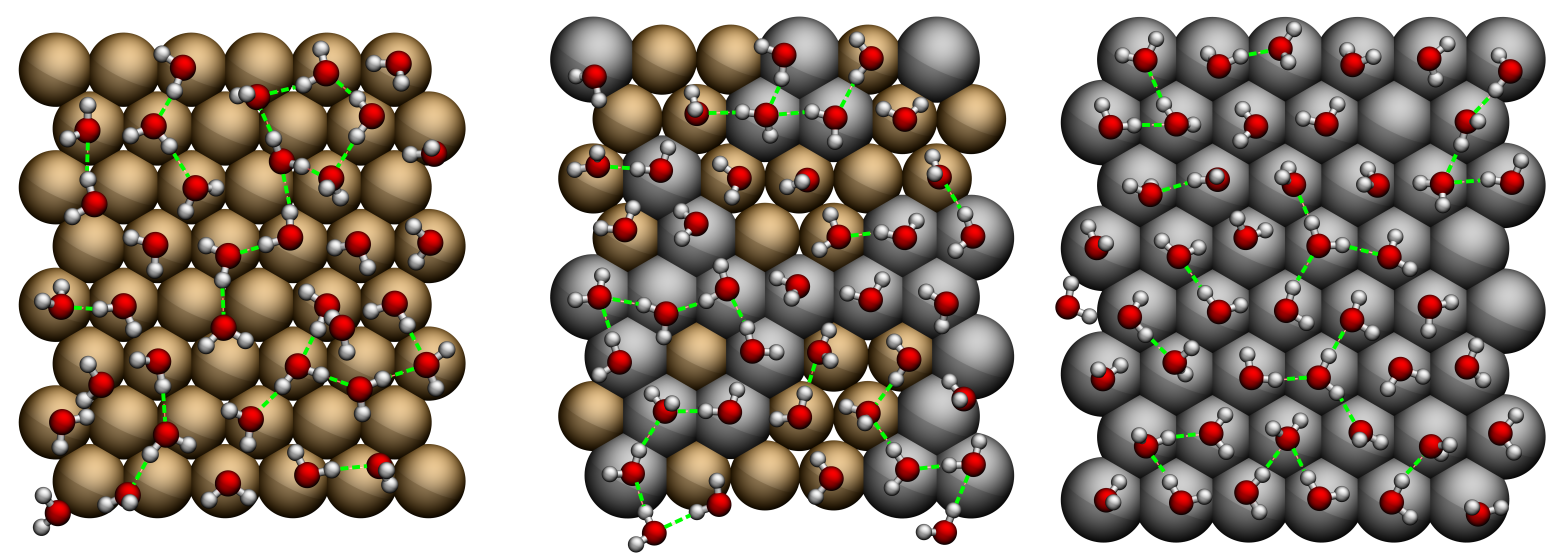

Figure 13: Snapshot extracted from molecular dynamcis of water layer on top of $\mathrm{Cu}$ (111) (a), $\mathrm{Cu}_{0.5} \mathrm{Ag}_{0.5}$ (111) (b) and $\mathrm{Ag}$ (111) (c). Cu is represented in brown, while $\mathrm{Ag}$ is shown in grey. Hydrogen bonds (as determined by default settings of VMD) are shown as dashed green lines.

The corresponding curves for the random $\mathrm{Au} / \mathrm{Pt}$ (111) alloy are provided in Fig. 14 to probe the influence of a contrasted adsorption while keeping a similar inter-metallic distance. In contrast with $\mathrm{Ag} / \mathrm{Cu}$, the $\mathrm{Au} / \mathrm{Pt}$ alloy shows a clear preference for water adsorption on top of the Pt atoms, which is in line with the observations for the monometallic surfaces (Fig. 11). Furthermore, the overall coverage is quite constant, again reflecting the behavior of $\operatorname{Pt}(111)$ and $\mathrm{Au}(111)$ which have similarly dense first layers. Despite the overall nearly constant behavior across the composition, there is a slight increase in the percentage of occupied Au top sites when increasing the Pt molar fraction. This can be rationalized via the templating effect of the surrounding Pt atoms, where water molecules 


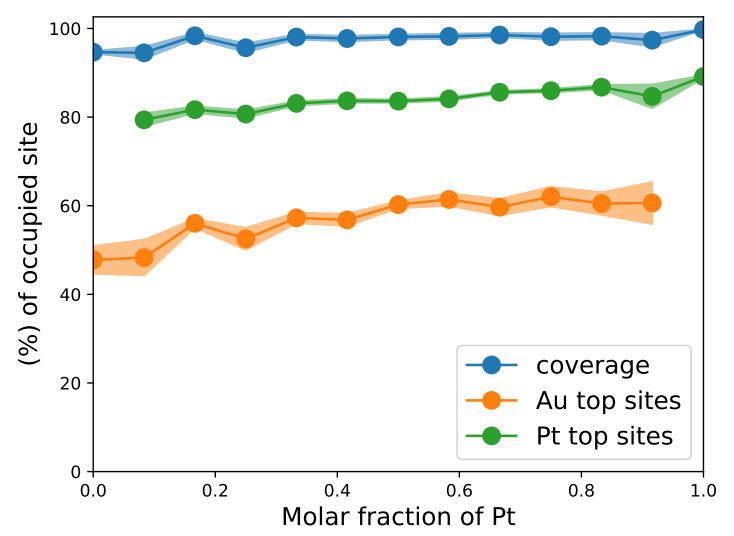

Figure 14: Percentage of the top sites of gold and platinum occupied by water molecule and global coverage of the surface for multiple alloy composition of $\mathrm{Au} / \mathrm{Pt}$. The coverage is defined as the number of oxygen atom in the first layer (between 0 and $4.5 \AA$ from the surface), divided by the total number of surface atoms. Confidence interval at $95 \%$ are represented as colored band surrounding the data. The corresponding variances were computed by block averaging. ${ }^{102}$

strongly prefer to adsorb on the top position and limit, therefore, the freedom of the water molecules in the vicinity of Au surface atoms. In terms of interface organization, the "constant" behavior of the $\mathrm{Au} / \mathrm{Pt}$ alloy hides the tuning from a disordered $\mathrm{Au}(111)$-like interface to a highly ordered $\operatorname{Pt}(111)$-like interface. This disordering is, however, visible when analyzing the angular distribution (Fig. 15) as a function of the Pt molar fraction. A shoulder at $\theta \approx 60^{\circ}$ appears when less than $\sim 30 \%$ of Pt are on the surface (Fig. 15a). Similarly, $\phi=0$ is lower for these Au rich surfaces at the benefit of $\phi>50^{\circ}$ adsorption modes, again attesting the higher disorder for Au rich interfaces. Even though subtle, such a tuning of the interface ordering could influence the solvation energy of molecules on the alloy surface.

In summary, our GAL19 simulations of alloy surfaces are among the first predictions of the interface organization as a function of the alloy composition, revealing subtle effects based on both geometric (lattice constant and its relation to the H-bonding distance) and intrinsic effects $\left(\mathrm{H}_{2} \mathrm{O}\right.$ binding preference). 


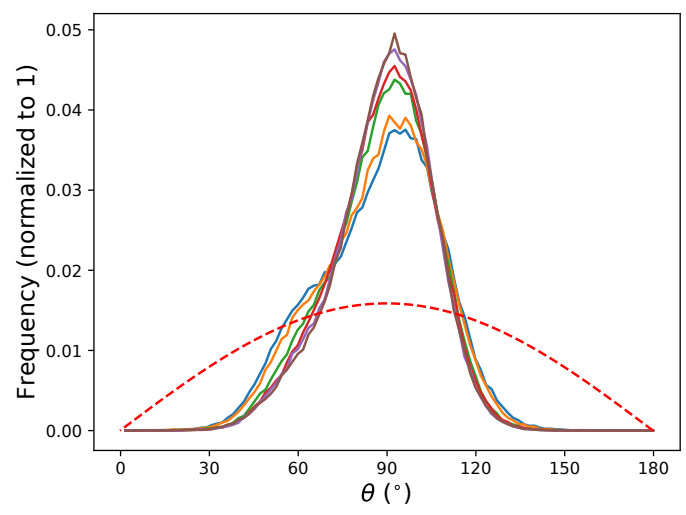

(a) $\theta$ Distribution

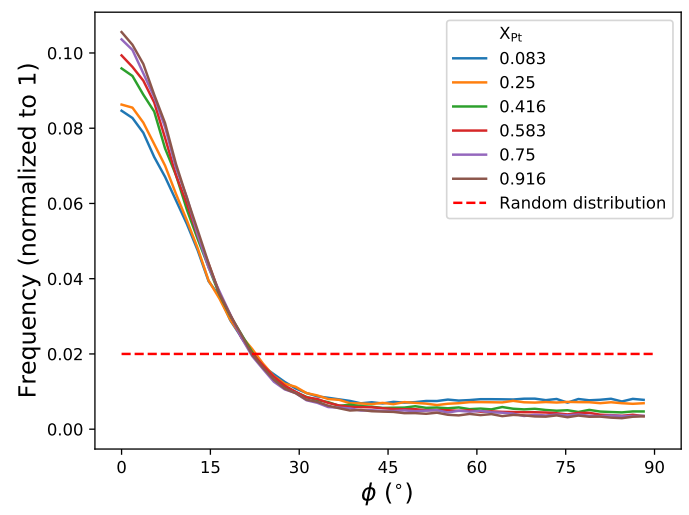

(b) $\phi$ Distribution

Figure 15: Angular distribution for different molar fraction of $\mathrm{Pt}\left(x_{P t}\right.$, given as a number in the legend) within the chemisorption layer (oxygen position $<4.5 \AA$ above the surface) of the (a) the cartwheel angle $\theta$ and (b) the propeller angle $\phi$ of the water molecules on PtAu alloys. The red dashed line represents an ideal random distribution of the dipoles. 


\section{Conclusion}

The key ingredient of the metal surface/water force field GAL19 is a locally defined surface normal computed based on atom pairwise sums. As a result of the pairwise nature, the novel force field is directly applicable to alloy surfaces. The 13 parameters have been fitted for 5 metals (Pt, Pd, Au, Ag, Cu) and two facets (111 and 100). For each metal and surface we have used a set of more than 600 configurations of one water molecule interacting with the surface computed at the DFT level. The parametrization leads to low average errors $\left(\mathrm{RMSD} \approx 1.0 \mathrm{kcal} \cdot \mathrm{mol}^{-1}\right)$ for the ten surfaces. Furthermore, the force field qualitatively reproduces the relative adsorption energy between the top and the hollow sites.

Molecular dynamics simulations of $1 \mathrm{~ns}$ for each surface show that the structuring of the metal/water interface does not depend a lot on the specific nature of the metal surface. The interface is composed of two layers, followed by the bulk structure of the liquid. The first layer is very dense, with mainly water molecules that are adsorbed flat on the metal surface. On Pt, most of the top sites are occupied while on Au only half of the top sites are occupied due to a lower corrugation between top and hollow sites. We have also studied random alloys, mixing either metals with similar intermetallic distance but contrasted adsorption energy $(\mathrm{Au} / \mathrm{Pt})$ or vice versa $(\mathrm{Ag} / \mathrm{Cu})$. Keeping in mind the limitations of GAL19 for alloys (absence of electronic effects), our results suggest that while the broad trends in properties of alloy/water interfaces (e.g., water coverages and top sites occupancy) can be inferred from the monometallic interfaces, the details might differ. For instance, when $\mathrm{Cu}$ is diluted in $\mathrm{Ag}$, it binds water more strongly since it is not limited anymore by the short $\mathrm{Cu}-\mathrm{Cu}$ intermetallic distance. Similarly, when $\mathrm{Au}$ is diluted in $\mathrm{Pt}$, water will tend to bind more to $\mathrm{Au}$ than when it is not as diluted, benefiting from the templating effect of the organized water layer on Pt.

GAL19 shows promising result for mono-molecular water adsorption, even though it does not include interfacial water-water many-body interactions which would be necessary in general. Similarly, the current parametrization is not adapted to ordered surface 
alloys. Nevertheless, in comparison to DFT, GAL19 allows a much more extensive phase space sampling of the noble metal/water interface. Therefore, our work opens the door to the evaluation of the solvation energy at the metal/water interface using a force field that captures the water-metal interaction with high fidelity.

\section{Acknowledgement}

The authors thank the SYSPROD project and AXELERA Pôle de Compétitivité for financial support (PSMN Data Center). This work benefited from the support of the project MuSiC ANR-14-CE06-0030 of the French National Research Agency (ANR). We are also grateful to the GdR SolvATE for a travel grant. Hatim Lagriai and Sana Abbassi have performed preliminary tests for the establishment of the DFT reference data. We thank Jinggang Lan for sharing the Pt/water AIMD trajectory from which we extracted snapshots.

\section{Supporting Information Available}

The supporting information contains: (i) the optimized force field parameters and additional tables and figures (pdf); (ii) an archive of all geometries of the training and test set (zip); (iii) a spreadsheet with raw-data for optimal top/hollow adsorptions (ods).

\section{References}

(1) Akpa, B. S.; D’Agostino, C.; Gladden, L. F.; Hindle, K.; Manyar, H.; McGregor, J.; Li, R.; Neurock, M.; Sinha, N.; Stitt, E. H. et al. Solvent effects in the hydrogenation of 2-butanone. J. Catal. 2012, 289, 30.

(2) Seh, Z. W.; Kibsgaard, J.; Dickens, C. F.; Chorkendorff, I.; Nørskov, J. K.; Jaramillo, T. F. 
Combining theory and experiment in electrocatalyis: Insights into materials design. Science 2017, 355, eaad4998.

(3) Shenghua, L.; He, Y.; Yuansheng, J. Lubrication Chemistry Viewed from DFT-Based Concepts and Electronic Structural Principles. Int. J. Mol. Sci. 2003, 5, 13.

(4) Gece, G. The use of quantum chemical methods in corrosion inhibitor studies. Corros. Sci. 2008, 50, 2981.

(5) Sinfelt, J. H. Catalysis by alloys and bimetallic clusters. Acc. Chem. Res. 1977, 10, 15-20.

(6) Wang, D.; Li, Y. Bimetallic Nanocrystals: Liquid-Phase Synthesis and Catalytic Applications. Adv. Mater. 2011, 23, 1044-1060.

(7) Matzdorf, C.; Grieve, A. US Patent, US 2019/0078179 A1.

(8) Toney, M. F.; Howard, J. N.; Richer, J.; Borges, G. L.; Gordon, J. G.; Melroy, O. R.; Wiesler, D. G.; Yee, D.; Sorensen, L. B. Voltage-dependent ordering of water molecules at an electrode-electrolyte interface. Nature 1994, 368, 444.

(9) Schiros, T.; Andersson, K. J.; Pettersson, L. G. M.; Nilsson, A.; Ogasawara, H. Chemical bonding of water to metal surfaces studied with core-level spectroscopies. J. Electron Spectrosc. Relat. Phenom. 2010, 177, 85.

(10) Velasco-Velez, J.-J.; Wu, C. H.; Pascal, T. A.; Wan, L. F.; Guo, J.; Prendergast, D.; Salmeron, M. The structure of interfacial water on gold electrodes studied by $\mathrm{x}$-ray absorption spectroscopy. Science 2014, 346, 831.

(11) Spohr, E.; Heinzinger, K. Molecular dynamics simulation of a water/metal interface. Chem. Phys. Letters 1986, 123, 218-221.

(12) Siepmann, J. I.; Sprik, M. Influence of surface topology and electrostatic potential on water/electrode systems. J. Chem. Phys. 1995, 102, 511-524. 
(13) Klesing, A.; Labrenz, D.; Santen, R. A. v. Ab initio simulation of 'liquid' water on a Pd surface. J. Chem. Soc., Faraday Trans. 1998, 94, 3229-3235, Publisher: The Royal Society of Chemistry.

(14) Izvekov, S.; Voth, G. A. Ab initio molecular dynamics simulation of the $\mathrm{Ag}(111)$-water interface. J. Chem. Phys. 2001, 115, 7196-7206.

(15) Heinz, H.; Vaia, R. A.; Farmer, B. L.; Naik, R. R. Accurate Simulation of Surfaces and Interfaces of Face-Centered Cubic Metals Using 12-6 and 9-6 Lennard-Jones Potentials. J. Phys. Chem. C 2008, 112, 17281-17290.

(16) Schnur, S.; Groß, A. Properties of metal-water interfaces studied from first principles. New J. of Phys. 2009, 11, 125003.

(17) Iori, F.; Di Felice, R.; Molinari, E.; Corni, S. GolP: An atomistic force-field to describe the interaction of proteins with $\mathrm{Au}(111)$ surfaces in water. J. Comput. Chem. 2009, 30, $1465-1476$.

(18) Limmer, D. T.; Willard, A. P.; Madden, P.; Chandler, D. Hydration of metal surfaces can be dynamically heterogeneous and hydrophobic. Proc. Natl. Acad. Sci. U. S. A. 2013, 110, 4200-4205.

(19) Sakong, S.; Forster-Tonigold, K.; Groß, A. The structure of water at a Pt(111) electrode and the potential of zero charge studied from first principles. J. Chem. Phys. 2016, $144,194701$.

(20) Le, J.; Iannuzzi, M.; Cuesta, A.; Cheng, J. Determining Potentials of Zero Charge of Metal Electrodes versus the Standard Hydrogen Electrode from Density-FunctionalTheory-Based Molecular Dynamics. Phys. Rev. Lett. 2017, 119, 016801.

(21) Steinmann, S. N.; Ferreira De Morais, R.; Götz, A. W.; Fleurat-Lessard, P.; Ian- 
nuzzi, M.; Sautet, P.; Michel, C. Force Field for Water over Pt(111): Development, Assessment, and Comparison. J. Chem. Theory Comput. 2018, 14, 3238-3251.

(22) Antony, A. C.; Liang, T.; Sinnott, S. B. Nanoscale Structure and Dynamics of Water on Pt and Cu Surfaces from MD Simulations. Langmuir 2018, 34, 11905-11911.

(23) Le, J.; Cuesta, A.; Cheng, J. The structure of metal-water interface at the potential of zero charge from density functional theory-based molecular dynamics. J. Electroanal. Chem. 2018, 819, 87-94.

(24) Gim, S.; Cho, K. J.; Lim, H.-K.; Kim, H. Structure, Dynamics, and Wettability of Water at Metal Interfaces. Sci. Rep. 2019, 9, 1-7.

(25) Mackerell, A. D. Empirical force fields for biological macromolecules: Overview and issues. J. Comput. Chem. 2004, 25, 1584-1604.

(26) Guo, N.; Caratzoulas, S.; Doren, D. J.; Sandler, S. I.; Vlachos, D. G. A perspective on the modeling of biomass processing. Energy Environ. Sci. 2012, 5, 6703-6716.

(27) Réocreux, R.; Michel, C. Rational design of heterogeneous catalysts for biomass conversion - Inputs from computational chemistry. Curr. Opin. Green Sustain. Chem. 2018, 10, 51-59.

(28) Xie, T.; Bodenschatz, C. J.; Getman, R. B. Insights into the roles of water on the aqueous phase reforming of glycerol. React. Chem. Eng. 2019, 4, 383-392.

(29) Michel, C.; Zaffran, J.; Ruppert, A. M.; Matras-Michalska, J.; Jedrzejczyk, M.; Grams, J.; Sautet, P. Role of water on metal catalyst performance for ketone hydrogenation. A join experimental and theoretical study on levulinic acid conversion into gamma-valerolactone. Chem. Comm. 2014, 50, 12450.

(30) Steinmann, S. N.; Michel, C.; Schwiedernoch, R.; Sautet, P. Impacts of electrode 
potentials and solvents on the electroreduction of $\mathrm{CO} 2$ : a comparison of theoretical approaches. Phys. Chem. Chem. Phys. 2015, 17, 13949-13963.

(31) Desai, S. K.; Pallassana, V.; Neurock, M. A Periodic Density Functional Theory Analysis of the Effect of Water Molecules on Deprotonation of Acetic Acid over Pd(111). J. Phys. Chem. B 2001, 105, 9171-9182.

(32) Michel, C.; Auneau, F.; Delbecq, F.; Sautet, P. C-H versus O-H Bond Dissociation for Alcohols on a Rh(111) Surface: A Strong Assistance from Hydrogen Bonded Neighbors. ACS Catal. 2011, 1, 1430-1440.

(33) Hibbitts, D. D.; Loveless, B. T.; Neurock, M.; Iglesia, E. Mechanistic Role of Water on the Rate and Selectivity of Fischer-Tropsch Synthesis on Ruthenium Catalysts. Angew. Chem. Int. Ed. 2013, 52, 12273-12278.

(34) Garcia-Rates, M.; Garcia-Muelas, R.; Lopez, N. Solvation Effects on Methanol Decomposition on $\operatorname{Pd}(111), \operatorname{Pt}(111)$, and $\operatorname{Ru}(0001)$. J. Phys. Chem. C 2017, 121, 13803-13809.

(35) Schweitzer, B.; Steinmann, S. N.; Michel, C. Can microsolvation effects be estimated from vacuum computations? A case-study of alcohol decomposition at the $\mathrm{H}_{2} \mathrm{O} / \mathrm{Pt}(111)$ interface. Phys. Chem. Chem. Phys. 2019, 21, 5368-5377.

(36) Behtash, S.; Lu, J.; Mamun, O.; Williams, C. T.; Monnier, J. R.; Heyden, A. Solvation Effects in the Hydrodeoxygenation of Propanoic Acid over a Model Pd(211) Catalyst. J. Phys. Chem. C 2016, 120, 2724-2736.

(37) Faheem, M.; Saleheen, M.; Lu, J.; Heyden, A. Ethylene glycol reforming on Pt(111): first-principles microkinetic modeling in vapor and aqueous phases. Catal. Sci. Technol. 2016, 6, 8242-8256.

(38) Gu, G. H.; Schweitzer, B.; Michel, C.; Steinmann, S. N.; Sautet, P.; Vlachos, D. G. 
Group Additivity for Aqueous Phase Thermochemical Properties of Alcohols on Pt(111). J. Phys. Chem. C 2017, 10.1021/acs.jpcc.7b07340.

(39) Steinmann, S. N.; Sautet, P. Assessing a First-Principles Model of an Electrochemical Interface by Comparison with Experiment. J. Phys. Chem. C 2016, 120, 5619-5623.

(40) Liu, J.; Cao, X.-M.; Hu, P. Density functional theory study on the activation of molecular oxygen on a stepped gold surface in an aqueous environment: a new approach for simulating reactions in solution. Phys. Chem. Chem. Phys. 2014, 16, 4176-4185.

(41) Bellarosa, L.; Garcia-Muelas, R.; Revilla-Lopez, G.; Lopez, N. Diversity at the WaterMetal Interface: Metal, Water Thickness, and Confinement Effects. ACS Cent. Sci. 2016, 2, 109-116.

(42) de Morais, R. F.; Kerber, T.; Calle-Vallejo, F.; Sautet, P.; Loffreda, D. Capturing Solvation Effects at a Liquid/Nanoparticle Interface by Ab Initio Molecular Dynamics: Pt201 Immersed in Water. Small 2016, 12, 5312-5319.

(43) Faheem, M.; Heyden, A. Hybrid Quantum Mechanics/Molecular Mechanics Solvation Scheme for Computing Free Energies of Reactions at Metal-Water Interfaces. J. Chem. Theory Comput. 2014, 10, 3354-3368.

(44) Steinmann, S. N.; Sautet, P.; Michel, C. Solvation free energies for periodic surfaces: comparison of implicit and explicit solvation models. Phys. Chem. Chem. Phys. 2016, $18,31850-31861$.

(45) Saleheen, M.; Heyden, A. Liquid-Phase Modeling in Heterogeneous Catalysis. ACS Catal. 2018, 8, 2188-2194.

(46) Staub, R.; Steinmann, S. N. Parameter-free coordination numbers for solutions and interfaces. J. Chem. Phys. 2020, 152, 024124. 
(47) Brenner, D. W. Empirical potential for hydrocarbons for use in simulating the chemical vapor deposition of diamond films. Phys. Rev. B 1990, 42, 9458-9471.

(48) Stuart, S. J.; Tutein, A. B.; Harrison, J. A. A reactive potential for hydrocarbons with intermolecular interactions. J. Chem. Phys. 2000, 112, 6472-6486.

(49) Brenner, D. W.; Shenderova, O. A.; Harrison, J. A.; Stuart, S. J.; Ni, B.; Sinnott, S. B. A second-generation reactive empirical bond order (REBO) potential energy expression for hydrocarbons. J. Phys.: Condens. Matter 2002, 14, 783-802.

(50) van Duin, A. C. T.; Dasgupta, S.; Lorant, F.; Goddard, W. A. ReaxFF: A Reactive Force Field for Hydrocarbons. J. Phys. Chem. A 2001, 105, 9396-9409.

(51) van Duin, A. C. T.; Strachan, A.; Stewman, S.; Zhang, Q.; Xu, X.; Goddard, W. A. ReaxFF $_{\text {siO }}$ Reactive Force Field for Silicon and Silicon Oxide Systems. J. Phys. Chem. A 2003, 107, 3803-3811.

(52) Naserifar, S.; Goddard, W. A. The quantum mechanics-based polarizable force field for water simulations. J. Chem. Phys. 2018, 149, 174502.

(53) Artrith, N.; Behler, J. High-dimensional neural network potentials for metal surfaces: A prototype study for copper. Phys. Rev. B 2012, 85.

(54) Staub, R.; Iannuzzi, M.; Khaliullin, R. Z.; Steinmann, S. N. Energy Decomposition Analysis for Metal Surface-Adsorbate Interactions by Block Localized Wave Functions. J. Chem. Theory Comput. 2019, 15, 265-275.

(55) Steinmann, S. N.; Fleurat-Lessard, P.; Götz, A. W.; Michel, C.; Ferreira de Morais, R.; Sautet, P. Molecular mechanics models for the image charge, a comment on "including image charge effects in the molecular dynamics simulations of molecules on metal surfaces". J. Comput. Chem. 2017, 38, 2127-2129. 
(56) Chen, W.; Schmidt, D.; Schneider, W. F.; Wolverton, C. Ordering and Oxygen Adsorption in Au-Pt/Pt(111) Surface Alloys. J. Phys. Chem. C 2011, 115, 17915-17924.

(57) Zhu, B.; Creuze, J.; Mottet, C.; Legrand, B.; Guesmi, H. CO Adsorption-Induced Surface Segregation and Formation of Pd Chains on AuPd(100) Alloy: Density Functional Theory Based Ising Model and Monte Carlo Simulations. J. Phys. Chem. C 2016, 120, 350-359.

(58) Vignola, E.; Steinmann, S. N.; Le Mapihan, K.; Vandegehuchte, B. D.; Curulla, D.; Sautet, P. Acetylene Adsorption on Pd-Ag Alloys: Evidence for Limited Island Formation and Strong Reverse Segregation from Monte Carlo Simulations. J. Phys. Chem. C 2018, 122, 15456-15463.

(59) Tang, K. T.; Toennies, J. P. An improved simple model for the van der Waals potential based on universal damping functions for the dispersion coefficients. J. Chem. Phys. 1984, 80, 3726-3741.

(60) Born, M.; Mayer, J. E. Zur Gittertheorie der Ionenkristalle. Z. Physik 1932, 75, 1-18.

(61) Buckingham, R. A.; Lennard-Jones, J. E. The classical equation of state of gaseous helium, neon and argon. Proc. R. Soc. A 1938, 168, 264-283.

(62) Steinmann, S. N.; Csonka, G.; Corminboeuf, C. Unified Inter- and Intramolecular Dispersion Correction Formula for Generalized Gradient Approximation Density Functional Theory. J. Chem. Theory Comput. 2009, 5, 2950-2958.

(63) Steinmann, S. N.; Corminboeuf, C. Comprehensive Benchmarking of a DensityDependent Dispersion Correction. J. Chem. Theory Comput. 2011, 7, 3567-3577.

(64) Zimbitas, G.; Haq, S.; Hodgson, A. The structure and crystallization of thin water films on Pt(111). J. Chem. Phys. 2005, 123, 174701. 
(65) Steinmann, S. N.; Corminboeuf, C. A generalized-gradient approximation exchange hole model for dispersion coefficients. J. Chem. Phys. 2011, 134, 044117.

(66) William L. Jorgensen,; Chandrasekhar, J.; Madura, J. D.; Impey, R. W.; Klein, M. L. Comparison of simple potential functions for simulating liquid water. J. Chem. Phys. $1983,79,926-935$.

(67) Soler, J. M.; Artacho, E.; Gale, J. D.; García, A.; Junquera, J.; Ordejón, P.; SánchezPortal, D. The SIESTA method for ab initio order- $N$ materials simulation. J. Phys.: Condens. Matter 2002, 14, 2745-2779.

(68) Kresse, G. Ab initio molecular dynamics for liquid metals. J. Non-Cryst. Solids 1995, 192-193, 222-229.

(69) Kresse, G.; Furthmüller, J. Efficient iterative schemes for ab initio total-energy calculations using a plane-wave basis set. Phys. Rev. B 1996, 54, 11169-11186.

(70) Perdew, J. P.; Wang, Y. Accurate and simple analytic representation of the electron-gas correlation energy. Phys. Rev. B 1992, 45, 13244-13249.

(71) Perdew, J. P.; Burke, K.; Ernzerhof, M. Generalized Gradient Approximation Made Simple. Phys. Rev. Lett. 1996, 77, 3865-3868.

(72) Gautier, S.; Steinmann, S. N.; Michel, C.; Fleurat-Lessard, P.; Sautet, P. Molecular adsorption at $\operatorname{Pt}(111)$. How accurate are DFT functionals? Phys. Chem. Chem. Phys. 2015, 17, 28921.

(73) Blöchl, P. E. Projector augmented-wave method. Phys. Rev. B 1994, 50, 17953-17979.

(74) Kresse, G.; Joubert, D. From ultrasoft pseudopotentials to the projector augmentedwave method. Phys. Rev. B 1999, 59, 1758-1775.

(75) Monkhorst, H. J.; Pack, J. D. Special points for Brillouin-zone integrations. Phys. Rev. B 1976, 13, 5188-5192. 
(76) Izadi, S.; Onufriev, A. V. Accuracy limit of rigid 3-point water models. J. Chem. Phys. 2016, $145,074501$.

(77) Dang, L. X.; Chang, T.-M. Molecular dynamics study of water clusters, liquid, and liquid-vapor interface of water with many-body potentials. J. Chem. Phys. 1997, 106, 8149-8159.

(78) Schütt, O.; Messmer, P.; Hutter, J.; VandeVondele, J. Electronic Structure Calculations on Graphics Processing Units; John Wiley \& Sons, Ltd, 2016; pp 173-190.

(79) Borstnik, U.; VandeVondele, J.; Weber, V.; Hutter, J. Sparse matrix multiplication: The distributed block-compressed sparse row library | Elsevier Enhanced Reader. Parallel Computing 2014, 40, 47-58.

(80) Hutter, J.; Iannuzzi, M.; Schiffmann, F.; VandeVondele, J. cp2k: atomistic simulations of condensed matter systems. WIREs Computational Molecular Science 2014, 4, 15-25.

(81) Frigo, M.; Johnson, S. The Design and Implementation of FFTW3. Proc. IEEE 2005, 93, 216-231.

(82) Essmann, U.; Perera, L.; Berkowitz, M. L.; Darden, T.; Lee, H.; Pedersen, L. G. A smooth particle mesh Ewald method. J. Chem. Phys. 1995, 103, 8577-8593.

(83) Nosé, S. A unified formulation of the constant temperature molecular dynamics methods. J. Chem. Phys. 1984, 81, 511-519.

(84) Nosé, S. A molecular dynamics method for simulations in the canonical ensemble. Molecular Physics 1984, 52, 255-268.

(85) Vegard, L. Die Konstitution der Mischkristalle und die Raumfüllung der Atome. Z. Physik 1921, 5, 17-26.

(86) Carrasco, J.; Klimeš, J.; Michaelides, A. The role of van der Waals forces in water adsorption on metals. J. Chem. Phys. 2013, 138, 024708. 
(87) Michaelides, A.; Ranea, V. A.; de Andres, P. L.; King, D. A. General Model for Water Monomer Adsorption on Close-Packed Transition and Noble Metal Surfaces. Phys. Rev. Lett. 2003, 90, 216102.

(88) Carrasco, J.; Michaelides, A.; Scheffler, M. Insight from first principles into the nature of the bonding between water molecules and $4 \mathrm{~d}$ metal surfaces. J. Chem. Phys. 2009, 130, 184707.

(89) Calle-Vallejo, F.; Martínez, J. I.; García-Lastra, J. M.; Sautet, P.; Loffreda, D. Fast Prediction of Adsorption Properties for Platinum Nanocatalysts with Generalized Coordination Numbers. Angew. Chem. Int. Ed. 2014, 53, 8316-8319.

(90) Wulff G., XXV. Zur Frage der Geschwindigkeit des Wachsthums und der Auflösung der Krystallflächen. Z. Kri. 1901, 34, 449.

(91) Carrasco, J.; Hodgson, A.; Michaelides, A. A molecular perspective of water at metal interfaces. Nat. Mater. 2012, 11, 667-674.

(92) Doering, D. L.; Madey, T. E. The adsorption of water on clean and oxygen-dosed $\mathrm{Ru}(011)$. Surf. Sci. 1982, 123, 305-337.

(93) Nie, S.; Feibelman, P. J.; Bartelt, N. C.; Thürmer, K. Pentagons and Heptagons in the First Water Layer on Pt(111). Phys. Rev. Lett. 2010, 105, 026102.

(94) Michel, C.; Goltl, F.; Sautet, P. Early stages of water/hydroxyl phase generation at transition metal surfaces - synergetic adsorption and $\mathrm{O}-\mathrm{H}$ bond dissociation assistance. Phys. Chem. Chem. Phys. 2012, 14, 15286-15290.

(95) Osawa, M.; Tsushima, M.; Mogami, H.; Samjeské, G.; Yamakata, A. Structure of Water at the Electrified Platinum-Water Interface: A Study by Surface-Enhanced Infrared Absorption Spectroscopy. J. Phys. Chem. C 2008, 112, 4248-4256. 
(96) Ataka, K.-i.; Yotsuyanagi, T.; Osawa, M. Potential-Dependent Reorientation of Water Molecules at an Electrode/Electrolyte Interface Studied by Surface-Enhanced Infrared Absorption Spectroscopy. J. Phys. Chem. 1996, 100, 10664-10672.

(97) Iwasita, T.; Xia, X. Adsorption of water at $\mathrm{Pt}(111)$ electrode in $\mathrm{HClO} 4$ solutions. The potential of zero charge. J. Electroanal. Chem. 1996, 411, 95-102.

(98) Duwez, P.; Willens, R. H.; Klement, W. Continuous Series of Metastable Solid Solutions in Silver-Copper Alloys. J. Appl. Phys. 1960, 31, 1136-1137.

(99) Tsaur, B. Y.; Lau, S. S.; Mayer, J. W. Continuous series of metastable Ag-Cu solid solutions formed by ion-beam mixing. Appl. Phys. Lett. 1980, 36, 823-826.

(100) Valodkar, M.; Modi, S.; Pal, A.; Thakore, S. Synthesis and anti-bacterial activity of $\mathrm{Cu}, \mathrm{Ag}$ and $\mathrm{Cu}-\mathrm{Ag}$ alloy nanoparticles: A green approach. Mater. Res. Bull. 2011, 46, 384-389.

(101) Dettelbach, K. E.; He, J.; Johnson, N. J. J.; Huang, A.; Bottomley, A.; Lam, B.; Salvatore, D. A.; Berlinguette, C. P. Kinetic phases of Ag-Cu alloy films are accessible through photodeposition. J. Mater. Chem. A 2019, 7, 711-715.

(102) Flyvbjerg, H.; Petersen, H. G. Error estimates on averages of correlated data. J. Chem. Phys. 1989, 91, 461-466.

(103) Luo, J.; Maye, M. M.; Petkov, V.; Kariuki, N. N.; Wang, L.; Njoki, P.; Mott, D.; Lin, Y.; Zhong, C.-J. Phase Properties of Carbon-Supported Gold-Platinum Nanoparticles with Different Bimetallic Compositions. Chem. Mater. 2005, 17, 3086-3091.

(104) Schrinner, M.; Proch, S.; Mei, Y.; Kempe, R.; Miyajima, N.; Ballauff, M. Stable Bimetallic Gold-Platinum Nanoparticles Immobilized on Spherical Polyelectrolyte Brushes: Synthesis, Characterization, and Application for the Oxidation of Alcohols. Adv. Mater. 2008, 20, 1928-1933. 
(105) Zhang, S.; Shao, Y.; Liao, H.-g.; Liu, J.; Aksay, I. A.; Yin, G.; Lin, Y. Graphene Decorated with PtAu Alloy Nanoparticles: Facile Synthesis and Promising Application for Formic Acid Oxidation. Chem. Mater. 2011, 23, 1079-1081. 
Graphical TOC Entry

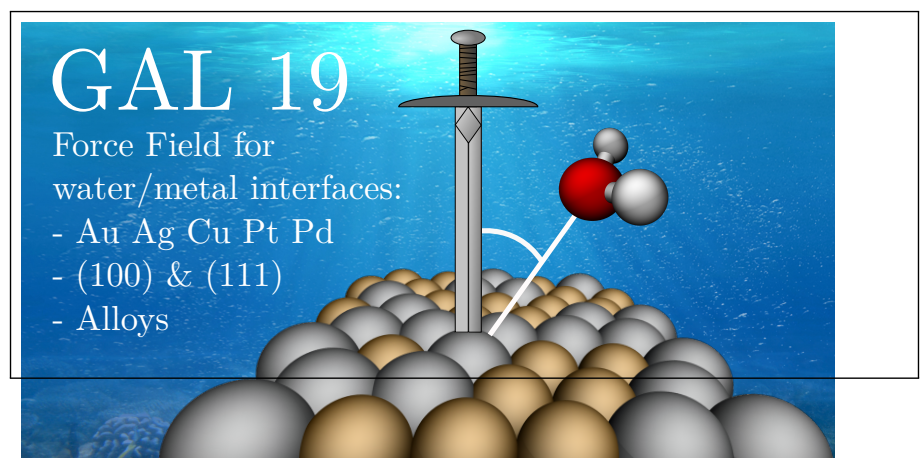

